\title{
How can young massive clusters reach their present-day sizes?
}

\author{
Sambaran Banerjee ${ }^{1,2}$ and Pavel Kroupa ${ }^{2}$ \\ 1 Argelander-Institut für Astronomie (AIfA), Auf dem Hügel 71, 53121 Bonn, Germany \\ e-mail: sambaran@astro.uni-bonn.de \\ ${ }^{2}$ Helmholtz-Instituts für Strahlen- und Kernphysik (HISKP), Nussallee 14-16, 53115 Bonn, Germany \\ Received 9 July 2015 / Accepted 13 October 2015
}

\begin{abstract}
Context. The classic question of how young massive star clusters attain the shapes and sizes, as we find them today, is still a difficult one. Both observational and computational studies of star-forming massive molecular gas clouds suggest that massive cluster formation is primarily triggered along the small-scale $(\lesssim 0.3 \mathrm{pc})$ filamentary substructures within the clouds.

Aims. The present study investigates the possible ways in which a filament-like, compact, massive star cluster (effective radius $0.1-0.3 \mathrm{pc})$ can expand more than 10 times, still remaining massive enough $\left(\gtrsim 10^{4} M_{\odot}\right)$ to become the young massive star cluster that we observe today.

Methods. To this end, model massive clusters (initially $10^{4}-10^{5} M_{\odot}$ ) are evolved using Sverre Aarseth's state-of-the-art $N$-body code NBODY7. Apart from the accurate calculation of two-body relaxation of the constituent stars, these evolutionary models take into account stellar-evolutionary mass loss and dynamical energy injection due to massive, tight primordial binaries and stellar-remnant black holes and neutron stars. These calculations also include a solar-neighbourhood-like external tidal field. All the computed clusters expand with time, and their sizes (effective radii) are compared with those observed for young massive clusters $(\leqslant 100 \mathrm{Myr})$ in the Milky Way and other nearby galaxies.

Results. In this study, it is found that beginning from the above compact sizes, a star cluster cannot expand on its own, i.e., due to two-body relaxation, stellar mass loss, and dynamical heating by primordial binaries and compact stars up to the observed sizes of young massive clusters; star clusters always remain much more compact than the observed ones.

Conclusions. This calls for additional mechanisms that boost the expansion of a massive cluster after its assembly. Using further $N$-body calculations, it is shown that a substantial residual gas expulsion with $\approx 30 \%$ star formation efficiency can indeed swell the newborn embedded cluster adequately. The limitations of the present calculations and their consequences are discussed.
\end{abstract}

Key words. galaxies: star clusters: general - methods: numerical - star: formation - stars: kinematics and dynamics

\section{Introduction}

Star clusters are being observed in our Milky Way and in all external galaxies with increasing detail. However, understanding how they form in the first place still poses a major challenge in astronomy. It is crucial to address this fundamental question because most, if not all, stars appear to form in embedded clusters (Elmegreen 1983; Lada \& Lada 2003). A key question in this regard is how the exposed clusters' parsec-scale, smooth, centrally pronounced, near spherical shape, observed at all ages $\gtrsim 1$ Myr can be explained. This is in sharp contrast with the irregular and much extended (10s of parsecs) morphology of molecular clouds where stellar nurseries form through gravitational collapse and fragmentation of dense substructures. A lack of an age range among the members of the youngest star clusters (see e.g. Bastian \& Silva-Villa 2013; Hollyhead et al. 2015) indicates that these stars form in a burst over a short period of time. This implies that short-timescale dynamical processes, like violent relaxation (Spitzer 1987), immediately follow or occur simultaneously with the formation of the proto-stars that shape the newly born cluster.

Recent unprecedented achievements of spatial and spectral resolution in IR and sub-mm wavelengths with instruments like Herschel and ALMA have revealed an intricate network of filamentary substructures in dense regions of molecular clouds (see André et al. 2014, for a review). Even ALMA observations of "dark" molecular clumps such as
"The Brick" (G0.253+0.016) have revealed fine filamentary structures in them that show signatures of continuing gravitational collapse (Rathborne et al. 2015). From such observations, it can generally be concluded that the individual filamentary overdensities and their junctions in molecular clouds are highly compact; they are of typical projected widths of 0.1-0.3 pc (André et al. 2011, 2014). Both theoretical and observational studies indicate that groups of stars or protostars preferentially form within the filaments and at filament junctions (André et al. 2014; Schneider et al. 2010, 2012; Tafalla \& Hacar 2014; Duarte-Cabral et al. 2011), giving rise to $\lesssim 0.5$ pc dense proto-stellar clumps (or pre-cluster cloud core; e.g. Lada \& Lada 2003; Traficante et al. 2015). Semi-analytic studies, e.g. Marks \& Kroupa (2012), also indicate similar gas clump sizes that depend weakly on their total mass. There is also evidence for the formation of massive star clusters triggered by collisions between massive molecular clouds (Furukawa et al. 2009; Fukui et al. 2014, 2016).

In Banerjee \& Kroupa (2015a), the role of violent relaxation in shaping a young cluster is studied in detail. It is demonstrated that in order to have a smooth-profiled, spherical cluster right from Myr-age as is seen in massive starburst clusters (see below), the stellar system involved in the aggregation process must be "near-monolithic". This implies the formation of either a single monolithic proto-cluster within a dense molecular clump or of several sub-clusters that merge in less than $\mathrm{Myr}$ 
from parsec-scale separations. In this case, the exact constraints can differ from cluster to cluster; in Banerjee \& Kroupa (2015a) the formation of the well-observed NGC 3603 young cluster is studied. Once a monolithic initial phase is attained, its evolution can be studied in realistic detail using direct $N$-body calculations, as is widely done in the literature (see e.g. Kroupa et al. 2001; Baumgardt \& Kroupa 2007; Pfalzner \& Kaczmarek 2013; Banerjee \& Kroupa 2014). Such studies often assume a star formation efficiency (SFE), $\epsilon$, implying the presence of primordial gas, embedding the initial stellar distribution. The gas is to be expelled by feedback from massive stars (Dale et al. 2015; Banerjee \& Kroupa 2015b) on a timescale comparable to the cluster's dynamical time which is mimicked by a time-decaying, cluster-centric external potential. While this approach definitely leads to oversimplification and idealization, it still captures the primary dynamical consequences. In particular, the studies have reproduced well the detailed observed properties of the ONC, the Pleiades, R136 and NGC 3603 young clusters (Kroupa et al. 2001; Banerjee \& Kroupa 2013, 2014).

These studies also imply that the initial mass and size of the embedded cluster and the corresponding clump SFE primarily determines the subsequent evolution of the stellar system. The rapidity of the gas expulsion is crucial as well. Observations of embedded systems in the solar neighbourhood (Lada \& Lada 2003) and hydrodynamical simulations of fragmentation of gas clouds and gas accretion onto proto-stars (Bate 2009, 2012; Bate et al. 2014), which include radiative feedback and magnetic fields, both indicate a maximum localized SFE of $\approx 30 \%$. These conditions are discussed in more detail in Sect. 3.

We now turn to the star clusters of the Milky Way and other nearby galaxies. In this study, we will primarily restrict ourselves to young massive star clusters (YMCs). A widely accepted definition of these objects (Portegies Zwart et al. 2010) is star clusters that have a present-day photometric mass exceeding $M_{\text {phot }} \gtrsim 10^{4} M_{\odot}$ and that are younger than $t \lesssim 100$ Myr by stellar age. These limits are not robust but are found to serve as good representative values. By stellar-evolutionary age, a $100 \mathrm{Myr}$-old stellar system is rather old since, by this time, all of its massive stars have evolved to produce their compact remnants. They are called "young" because of their dynamical age, since their age is $\approx 10-100$ times their present-day dynamical time (crossing time at half-mass radius), $\tau_{\mathrm{cr}}$. Globular clusters, on the other hand, are of age $t \gtrsim 1000 \tau_{\mathrm{cr}}$, and are designated as dynamically old. We note that more massive and concentrated clusters will be dynamically older at a given stellar age since they have shorter $\tau_{\mathrm{cr}}$. On the other hand, if the stellar system is dynamically too young, for example $t \lesssim 5 \tau_{\text {cr }}$, then phenomenologically it may or may not remain gravitationally bound through its future evolution. We call such gas-free dynamically young systems simply "associations", as in Portegies Zwart et al. (2010). The YMCs, on the other hand, are truly gravitationally bound objects.

A number of YMCs and associations are observed in the Milky Way, the Local Group, and in other nearby external galaxies. A comprehensive compilation of these can be found in the review by Portegies Zwart et al. (2010). In our paper, we refer to this compilation in order to gain insight on the birth conditions of YMCs. This assortment of YMCs gives, in particular, a comprehensive picture of their sizes in our galaxies and in nearby galaxies. The sizes of star clusters and the implications for their birth conditions has always been a topic of interest and debate (Pfalzner 2009; Marks \& Kroupa 2012; Pfalzner \& Kaczmarek 2013; Pfalzner et al. 2014). A relatively unambiguous measure of a star cluster's size is its effective radius, $r_{\text {eff }}$, defined as the projected radius from its center containing half of the cluster's total bolometric luminosity. This is essentially the cluster's halfmass radius in projection. If the cluster is in virial equilibrium, $r_{\text {eff }}$ is also representative of the cluster's virial radius. A widely-used measure of the cluster's central region is its core radius, $r_{\mathrm{c}}$, of which multiple definitions are adopted in the literature (Heggie \& Hut 2003); a commonly used measure is the radial distance at which the surface luminosity density becomes half of its central value. The overall spatial scale of the stellar distribution is, of course, represented by its half-mass radius or effective radius, $r_{\text {eff }}$.

Table 1 enlists age $t$, total photometric mass $M_{\text {phot }}$, and effective radius $r_{\text {eff }}$ of YMCs in the Milky Way (hereafter MW), the Local Group (hereafter LG), and other nearby galaxies (hereafter OG) from Portegies Zwart et al. (2010). Figure 1 shows the histograms of $r_{\mathrm{eff}}, M_{\mathrm{phot}}$, and $t$ for the MW, LG, and OG YMCs in Table 1. In each panel of this figure, the distributions corresponding to the MW members are seen to be concentrated towards lower values and are narrower. In particular, the $M_{\text {phot }}$ ranges are similar for MW and LG members and extend to much larger values for OG clusters. The $r_{\text {eff }}$ and age distributions of LG and OG YMCs extend to much larger values than the MW ones. These trends can be explained by the fact that clusters with larger $r_{\mathrm{eff}}$ survive longer in the weaker tidal fields of the LMC, SMC, and other MW satellite galaxies and the fact that the starburst OGs (NGC 4038 and NGC 4449; see Table 1) host much more massive YMCs (super star clusters with $M_{\text {phot }} \gtrsim 10^{5} M_{\odot}$; hereafter SSCs) due to higher star formation activity in them. These SSCs potentially survive longer even in the more fluctuating and disruptive tidal fields of starburst and merging galaxies (see e.g. Renaud et al. 2015). Some of the widest clusters are also consistent with them being an ensemble of several YMCs (see Sect. 3). A detailed discussion of the differences in the YMCs of the MW and external galaxies is beyond the scope of this paper; see also Portegies Zwart et al. (2010) for a discussion.

Table 2 enlists age, $M_{\text {phot }}$, and $r_{\text {eff }}$ of the MW, LG, and OG associations from Portegies Zwart et al. (2010), and Fig. 2 shows their combined histograms. Overall, the associations are much lighter, larger, and younger than the YMCs (see above), which makes their $\tau_{\mathrm{cr}}$ longer and closer to their stellar ages, thereby making them only candidates for future bound clusters. For the comparisons used in this study, we will primarily consider the YMCs which are "actual" star clusters in the sense that a significant fraction of their mass is gravitationally self-bound at their present age. The filled symbols in the upper panel of Fig. 3 are the YMCs from Table 1 in the $r_{\text {eff }}-t$ plane, and those in the lower panel represent this plane for the associations from Table 2. These observed points and the computed curves (see Sect. 2) in the $r_{\mathrm{eff}}-t$ plane are colour-coded according to their corresponding bound masses ( $M_{\mathrm{phot}}$ for the data points and $M_{\mathrm{cl}}$ for the computed curves) at age $t$ (the common colour scale at the right of the panels). We shall continue to use this data in the subsequent figures as our primary observational reference for YMCs. In Fig. 3 (top panel), the $r_{\text {eff }}$ values of the YMCs seem to follow a moderate increasing trend with age. The youngest among them $(t \lesssim 4.5 \mathrm{Myr})$ that contain massive O-type stars are generally referred to as starburst clusters. The overall trend indicates a common evolutionary origin of these YMCs. Notably, Ryon et al. (2015) present HST/WFC3 measurements of YMCs in the M83 spiral galaxy using archival data. The mean and the distribution of $r_{\text {eff }} \mathrm{s}$ of these YMCs are qualitatively similar to Fig. 1 (top panel) and these $r_{\text {eff }}$ values also show an increasing trend with age.

In this work, we show how the two key observed parameters of YMCs, viz., the effective radius $r_{\text {eff }}$ and the present day mass 
S. Banerjee and P. Kroupa: Star cluster expansion

Table 1. A list of stellar age $t$, photometric mass $M_{\text {phot }}$, and effective radius $r_{\text {eff }}$ of bound star clusters, of age $t \lesssim 100$ Myr (young) and photometric mass $M_{\text {phot }} \gtrsim 10^{4} M_{\odot}$ (massive), observed in the Milky Way Galaxy, the Local Group galaxies, and other external galaxies.

\begin{tabular}{|c|c|c|c|c|}
\hline Galaxy name & Cluster name & Age $t$ (Myr) & $\log _{10}\left(M_{\text {phot }} / M_{\odot}\right)$ & $r_{\mathrm{eff}}(\mathrm{pc})$ \\
\hline Milky Way & Arches & $2.00(+2.00)$ & 4.30 & 0.40 \\
\hline Milky Way & DSB2003 & 3.50 & 3.80 & 1.20 \\
\hline Milky Way & NGC 3603 & $2.00(-1.00)$ & 4.10 & 0.70 \\
\hline Milky Way & Quintuplet & 4.00 & 4.00 & 2.00 \\
\hline Milky Way & RSGC01 & 12.00 & 4.50 & 1.50 \\
\hline Milky Way & RSGC02 & 17.00 & 4.60 & 2.70 \\
\hline Milky Way & RSGC03 & 18.00 & 4.50 & 5.00 \\
\hline Milky Way & Trumpler14 & 2.00 & 4.00 & 0.50 \\
\hline Milky Way & $\mathrm{Wd} 1$ & 3.50 & 4.50 & 1.00 \\
\hline Milky Way & $\mathrm{Wd} 2$ & 2.00 & 4.00 & 0.80 \\
\hline Milky Way & hPer & 12.80 & 4.20 & 2.10 \\
\hline Milky Way & XPer & 12.80 & 4.10 & 2.50 \\
\hline LMC & R136 & 3.0 & 4.78 & 1.70 \\
\hline LMC & NGC 1818 & 25.1 & 4.42 & 5.39 \\
\hline LMC & NGC 1847 & 26.3 & 4.44 & 32.58 \\
\hline LMC & NGC 1850 & 31.6 & 4.86 & 11.25 \\
\hline LMC & NGC 2004 & 20.0 & 4.36 & 5.27 \\
\hline LMC & NGC 2100 & 15.8 & 4.36 & 4.41 \\
\hline LMC & NGC 2136 & 100.0 & 4.30 & 3.42 \\
\hline LMC & NGC 2157 & 39.8 & 4.31 & 5.39 \\
\hline LMC & NGC 2164 & 50.1 & 4.18 & 4.76 \\
\hline LMC & NGC 2214 & 39.8 & 4.03 & 8.13 \\
\hline LMC & NGC 1711 & 50.1 & 4.24 & 5.19 \\
\hline M31 & KW246 & 75.9 & 4.19 & 3.20 \\
\hline M31 & B257D & 79.4 & 4.45 & 15.14 \\
\hline M31 & B318 & 70.8 & 4.38 & 6.61 \\
\hline M31 & B327 & 50.1 & 4.38 & 4.47 \\
\hline M31 & B448 & 79.4 & 4.58 & 16.22 \\
\hline M31 & Vdb0 & 25.1 & 4.85 & 7.40 \\
\hline M31 & KW 044 & 58.9 & 4.59 & 10.00 \\
\hline M31 & KW 120 & 87.1 & 4.57 & 2.60 \\
\hline M31 & KW 208 & 56.2 & 4.01 & 2.90 \\
\hline M31 & KW 272 & 53.7 & 4.50 & 9.00 \\
\hline M31 & B015D & 70.8 & 4.76 & 16.60 \\
\hline M31 & B040 & 79.4 & 4.50 & 12.88 \\
\hline M31 & B043 & 79.4 & 4.43 & 3.98 \\
\hline M31 & B066 & 70.8 & 4.25 & 6.76 \\
\hline NGC 6822 & HubbleIV & 25.1 & 4.00 & 2.00 \\
\hline SMC & NGC 330 & 25.1 & 4.56 & 6.11 \\
\hline ESO338IG & 23 & 7.08 & 6.70 & 5.20 \\
\hline M51 & $3 \mathrm{cl}-\mathrm{a}$ & 15.85 & 5.04 & 5.20 \\
\hline M51 & $3 \mathrm{cl}-\mathrm{b}$ & 5.01 & 5.91 & 2.30 \\
\hline M51 & a1 & 5.01 & 5.47 & 4.20 \\
\hline M82 & MGG9 & 9.55 & 5.92 & 2.60 \\
\hline M82 & A1 & 6.31 & 5.82 & 3.00 \\
\hline M82 & $\mathrm{F}$ & 60.26 & 6.70 & 2.80 \\
\hline
\end{tabular}

Notes. This list is compiled from Portegies Zwart et al. (2010; see their Tables 2-4) where the references for the data of the individual clusters are given. All these stellar systems are $\gtrsim 10$ times older than their present dynamical crossing time, $\tau_{\mathrm{cr}}$, which is a phenomenological criterion to identify them as gravitationally bound objects (see Portegies Zwart et al. 2010). 
Table 1. continued.

\begin{tabular}{lllll}
\hline \hline Galaxy name & Cluster name & Age $t(\mathrm{Myr})$ & $\log _{10}\left(M_{\text {phot }} / M_{\odot}\right)$ & $r_{\text {eff }}(\mathrm{pc})$ \\
\hline NGC 1140 & 1 & 5.01 & 6.04 & 8.00 \\
NGC 1487 & 2 & 8.51 & 5.20 & 1.20 \\
NGC 1487 & 1 & 8.32 & 5.18 & 2.30 \\
NGC 1487 & 3 & 8.51 & 4.88 & 2.10 \\
NGC 1569 & A & 12.02 & 6.20 & 2.30 \\
NGC 1569 & C & 3.02 & 5.16 & 2.90 \\
NGC 1569 & B & 19.95 & 5.74 & 2.10 \\
NGC 1569 & 30 & 91.20 & 5.55 & 2.50 \\
NGC 1705 & 1 & 15.85 & 5.90 & 1.60 \\
NGC 4038 & S2-1 & 8.91 & 5.47 & 3.70 \\
NGC 4038 & W99-1 & 8.13 & 5.86 & 3.60 \\
NGC 4038 & W99-16 & 10.00 & 5.46 & 6.00 \\
NGC 4038 & W99-2 & 6.61 & 6.42 & 8.00 \\
NGC 4038 & W99-15 & 8.71 & 5.70 & 1.40 \\
NGC 4038 & S1-1 & 7.94 & 5.85 & 3.60 \\
NGC 4038 & S1-2 & 8.32 & 5.70 & 3.60 \\
NGC 4038 & S1-5 & 8.51 & 5.48 & 0.90 \\
NGC 4038 & $2000-1$ & 8.51 & 6.23 & 3.60 \\
NGC 4038 & S2-2 & 8.91 & 5.60 & 2.50 \\
NGC 4038 & S2-3 & 8.91 & 5.38 & 3.00 \\
NGC 4449 & N-1 & 10.96 & 6.57 & 16.90 \\
NGC 4449 & N-2 & 3.02 & 5.00 & 5.80 \\
NGC 5236 & 805 & 12.59 & 5.29 & 2.80 \\
NGC 5236 & 502 & 100.00 & 5.65 & 7.60 \\
NGC 5253 & I & 11.48 & 5.38 & 4.00 \\
NGC 5253 & VI & 10.96 & 4.93 & 3.10 \\
NGC 6946 & 1447 & 11.22 & 5.64 & 10.00 \\
\hline
\end{tabular}

$M_{\text {phot }}$ can be used in combination to constrain the birth conditions of YMCs. Section 2 presents $N$-body calculations of secular size and mass evolution of model clusters beginning from compact initial conditions, which are compared with the clusters of Portegies Zwart et al. (2010). The approach for the numerical calculations are described in Sect. 2.1. These computations suggest the necessity of non-secular (i.e. by physical processes that are not inherent to the cluster) expansion of newborn clusters and these model calculations are presented in Sect. 3. In Sect. 4, the calculations are discussed in the context of the widely debated hierarchical merging scenario of YMCs. Concluding remarks, based on some recent notable studies, are presented in Sect. 5.

\section{Secular expansion of star clusters}

A possible way in which a star cluster can appear within a filamentary structure of molecular gas is through a localized but intense starburst at a privileged location in the filament, for example at a junction of multiple filaments. Such a location is prone to reach a much higher SFE than that for the whole cloud of a few percent owing to lateral contraction of the filaments and ample gas supply through them. The nascent cluster of protostars that would form this way would also be of the lateral subparsec scale length of the filaments, i.e. $\lesssim 0.3$ pc (see Sect. 1 and references therein). A cluster with $r_{\text {eff }} \lesssim 0.3$ pc is much more compact than the presently observed YMCs, as seen in Fig. 3. The most important question here is whether such a compact star cluster can expand on its own through its secular evolution to attain the presently observed sizes.

To reach the present-day sizes, such a compact cluster has to expand by a factor of $\gtrsim 10$. For a young massive cluster, multiple physical effects contribute to its secular expansion. As long as the massive stars remain on the main sequence (until $t \approx 4.5 \mathrm{Myr}$ ), mass loss due to stellar winds is the primary driver of the expansion. The most massive O-stars remain segregated (either primordially or dynamically) at the cluster's center (see below), so that the resulting central mass loss due to their strong winds results in a substantial expansion of the cluster. When the most massive stars begin to undergo supernova explosions, the central mass loss becomes more severe, causing a higher rate of expansion. This stellar mass loss dominated expansion continues for the first $\approx 50$ Myr.

After this stellar mass loss phase, the expansion of the cluster continues to be driven by dynamical heating due to the centrally segregated black holes (BHs) and neutron stars (NSs). Once the massive stars are reduced to BHs and NSs by their TypeII supernovae and core collapse, these remnants become the most massive members of the system, causing them to segregate strongly and to concentrate near the cluster's center. Their high density allows binaries to form via the three-body mechanism 

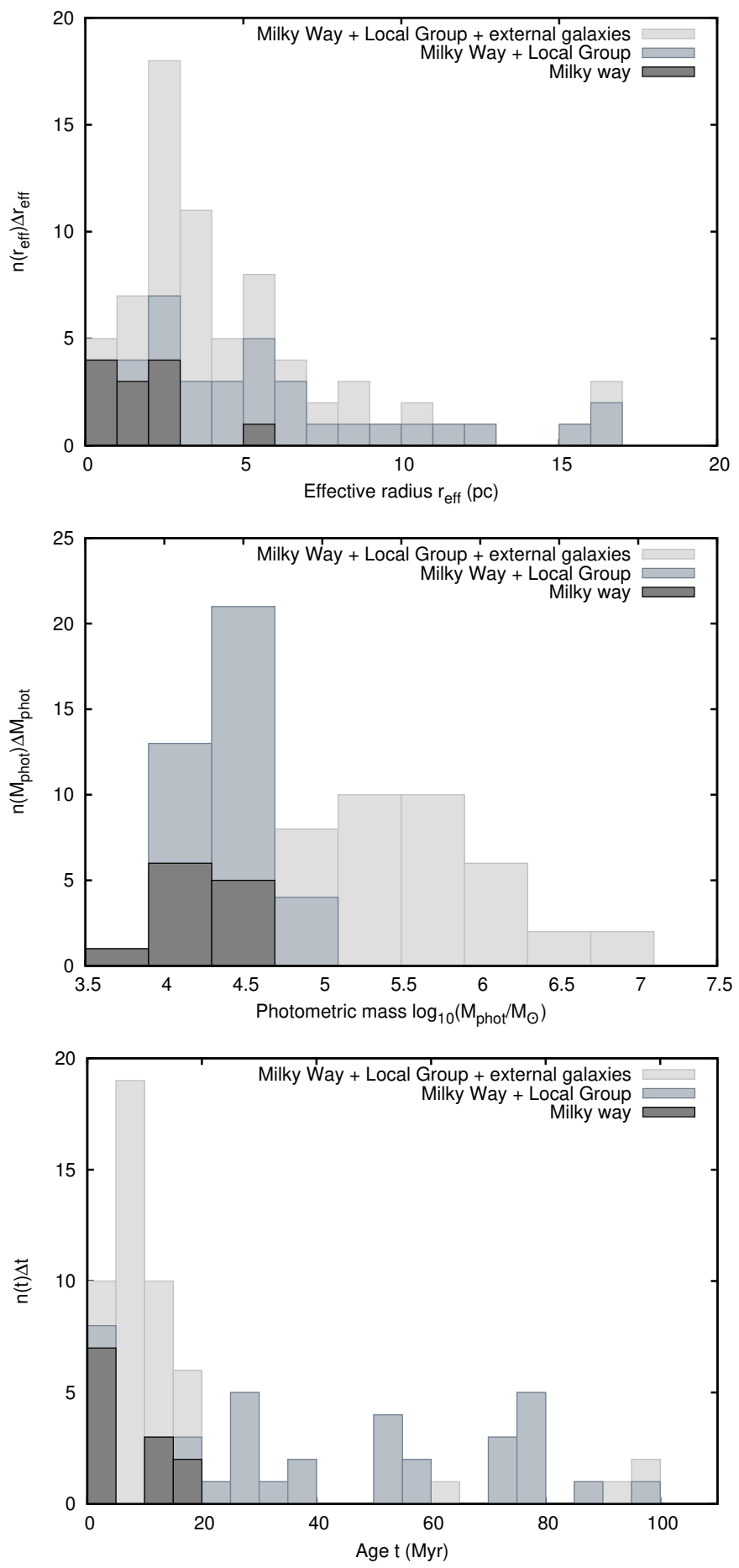

Fig. 1. Histograms of effective radius $r_{\text {eff }}$ (top panel), photometric mass $M_{\text {phot }}$ (middle), and stellar age $t$ (bottom) for young massive bound star clusters (YMCs) in the Milky Way, the Local Group, and in external galaxies. The data are from Table 1 . In each panel the clusters from the Milky Way, the Local Group, and external galaxies are cumulatively added to construct three distributions which are shaded differently.

(Heggie \& Hut 2003) which continue to undergo dynamical encounters. The energy generated in encounters with the binaries (known as "binary heating"; Heggie 1975; Heggie \& Hut 2003) heats and expands the cluster. The BHs, most which are of $>10 M_{\odot}$, are typically centrally concentrated within a fraction of a parsec and are most efficient at dynamically heating the cluster (Mackey et al. 2008; Banerjee et al. 2010; Banerjee 2011; Morscher et al. 2013). Once the majority of the BHs are depleted from the cluster through ejections due to dynamical encounters, the NSs take over (Aarseth 2012). The primary uncertainty in this process is the number of BHs and NSs that are formed with low enough natal kicks and are initially retained in the cluster. In the idealistic case, where all of them are retained at birth, the BHs and NSs can contribute to dynamically expanding the cluster for several $100 \mathrm{Myr}$.

Young star clusters are found to contain a significant fraction $(\gtrsim 70 \%)$ of tight primordial binaries of massive main-sequence stars (Sana \& Evans 2011, see also Sect. 2.1). These primordial binaries also inject energy into the cluster and contribute to its expansion through binary-single and binary-binary encounters and the associated binary heating and ejections of massive stars (Heggie \& Hut 2003; Banerjee \& Kroupa 2012; Banerjee et al. 2012; Oh et al. 2015).

Finally, it should be kept in mind that any gravitationally bound star cluster with a realistic stellar mass distribution can, in principle, expand purely due to two-body relaxation (i.e. even in the absence of stellar-evolutionary mass loss). This happens in a self-similar manner (half-mass radius, $r_{\mathrm{h}} \propto t^{2 / 3}$ ) where the cluster's two-body relaxation time is a fixed multiple of the cluster age (Hènon 1965). The self-similar expansion commences when the most massive particles (stars) sink to the cluster's center via dynamical friction causing an early core collapse. This in turn strongly concentrates the massive particles towards the cluster's center and they cause the cluster to expand via dynamical heating (see above). This heating and the expansion would, in fact, be more efficient than those due to stellar-mass BHs (see above) since the cluster's most massive stars are $\lesssim 10$ times heavier than the remnant BHs. As demonstrated in Fig. 1 of Gieles et al. (2012), which is for low-mass clusters of $N=256$ particles, the self-similar expansion commences only after the $r_{\mathrm{h}}(t)$ curve reaches the self-similar expansion line, $r_{\mathrm{h}}(t) \propto t^{2 / 3}$. For the computations for YMCs (see Sect. 2.1), which are much more massive $\left(N \gtrsim 17000,0.1 \lesssim r_{\mathrm{h}} \lesssim 0.3 \mathrm{pc}\right)$, the $r_{\mathrm{h}}(t)$ curves would begin far above the self-similar expansion line (see Fig. 1 in Gieles et al. 2012). In this case, stellar-evolutionary mass loss would take over the cluster expansion well before the self-similar expansion due to two-body relaxation can begin.

\subsection{N-body computations}

The dynamical relaxation of a self-gravitating many-body system is most realistically calculated using star-by-star direct $N$-body integration. In this work, we use the state-of-the-art $N$-body code, NBODY7 (formerly NBODY6; Aarseth 2003, 2012), to compute the secular evolution of star clusters. In addition to integrating the individual stars' orbits using the highly accurate fourth-order Hermite scheme and dealing with the diverging gravitational forces, for example during close encounters and hard binaries through two-body and many-body regularizations, NBODY7 also employs the well-tested analytical stellar and binary evolution recipes of Hurley et al. (2000, 2002), i.e. the SSE and the BSE schemes. Furthermore, mergers among stars and remnants are included through analytic recipes. Most importantly, no softening of gravitational forces is employed at any stage, ensuring that the energetics of close encounters, which plays a key role in the structural evolution of a star cluster, is accurately calculated. This numerical suite, therefore, naturally and realistically incorporates all the physical processes that contribute to the secular evolution and expansion of a star cluster (see above).

To address the question of how newborn star clusters can reach their present-day sizes from their highly compact dimensions at birth, we compute the evolution of a series of 
Table 2. A list of stellar age $t$, photometric mass $M_{\text {phot }}$, and effective radius $r_{\text {eff }}$ of young, massive (see Table 1; text) stellar associations observed in the Milky Way Galaxy, the Local Group of galaxies, and other external galaxies.

\begin{tabular}{|c|c|c|c|c|}
\hline Galaxy name & Cluster name & Age $t$ (Myr) & $\log _{10}\left(M_{\text {phot }} / M_{\odot}\right)$ & $r_{\mathrm{eff}}(\mathrm{pc})$ \\
\hline Milky Way & CYgOB & 2.5 & 4.40 & 5.20 \\
\hline Milky Way & IC 1805 & 2.00 & 4.20 & 12.50 \\
\hline Milky Way & ILac1 & 14.00 & 3.40 & 20.70 \\
\hline Milky Way & LowerCenCrux & 11.50 & 3.30 & 15.00 \\
\hline Milky Way & NGC 2244 & 2.00 & 3.90 & 5.60 \\
\hline Milky Way & NGC 6611 & 3.00 & 4.40 & 5.90 \\
\hline Milky Way & NGC 7380 & 2.00 & 3.80 & 6.50 \\
\hline Milky Way & ONC & 1.00 & 3.65 & 2.00 \\
\hline Milky Way & OriIa & 11.40 & 3.70 & 16.60 \\
\hline Milky Way & Orilb & 1.70 & 3.60 & 6.30 \\
\hline Milky Way & OriIc & 4.60 & 3.80 & 12.50 \\
\hline Milky Way & UpperCenCrux & 14.50 & 3.60 & 22.10 \\
\hline Milky Way & USco & 5.50 & 3.50 & 14.20 \\
\hline M31 & KW 249 & 5.0 & 4.30 & 13.50 \\
\hline M31 & KW 258 & 5.0 & 4.05 & 3.40 \\
\hline M33 & NGC 595 & 4.0 & 4.50 & 26.90 \\
\hline M33 & NGC 604 & 3.5 & 5.00 & 28.40 \\
\hline SMC & NGC 346 & 3.0 & 5.60 & 9.00 \\
\hline NGC 2403 & $\mathrm{I}-\mathrm{B}$ & 6.03 & 4.82 & 26.30 \\
\hline NGC 2403 & $\mathrm{I}-\mathrm{C}$ & 6.03 & 4.42 & 19.60 \\
\hline NGC 2403 & I-A & 6.03 & 5.06 & 20.60 \\
\hline NGC 2403 & II & 4.47 & 5.35 & 11.80 \\
\hline NGC 2403 & IV & 4.47 & 5.07 & 30.00 \\
\hline NGC 4214 & VI & 10.96 & 4.93 & 35.90 \\
\hline NGC 4214 & V & 10.96 & 5.73 & 83.90 \\
\hline NGC 4214 & VII & 10.96 & 5.33 & 40.40 \\
\hline NGC 4214 & I-A & 3.47 & 5.44 & 16.50 \\
\hline NGC 4214 & I-B & 3.47 & 5.40 & 33.00 \\
\hline NGC 4214 & I-D & 8.91 & 5.30 & 15.30 \\
\hline NGC 4214 & II-C & 2.00 & 4.86 & 21.70 \\
\hline NGC 5253 & IV & 3.47 & 4.72 & 13.80 \\
\hline
\end{tabular}

Notes. This list is compiled from Portegies Zwart et al. (2010; see their Tables 2-4) where the references for the data of the individual clusters are given. All these stellar systems are $\lesssim 5$ times younger than their present dynamical crossing time, $\tau_{\mathrm{cr}}$ (i.e. they are dynamically young), which implies that they may or may not be gravitationally bound objects.

model clusters using NBODY7. The initial conditions of the computed model clusters are given in Table 3; all of them initially have Plummer profiles (Plummer 1911) with half-mass radius, $r_{\mathrm{h}}(0) \approx 0.3 \mathrm{pc}$, and $1.0 \mathrm{pc}$ and are located at $R_{\mathrm{G}} \approx 8 \mathrm{kpc}$ Galactocentric distance (the solar distance) and they orbit the Galactic center at $V_{\mathrm{G}} \approx 220 \mathrm{~km} \mathrm{~s}^{-1}$. All computed clusters have a canonical initial mass function (IMF, Kroupa 2001) for the stellar members at zero-age main sequence (ZAMS) with an optimal sampling of the stellar masses, i.e. the most massive star in a cluster correlates with the initial cluster mass (Weidner \& Kroupa 2004; Weidner et al. 2013). The initial masses, $M_{\mathrm{cl}}(0)$, of the models are taken between $10^{4}-10^{5} M_{\odot}$. While this mass range is appropriate for the MW and LG YMCs, the OG YMCs are 10-100 times more massive. However, direct $\mathrm{N}$-body calculations for systems over the OG mass range are prohibitive using the presently available technology and therefore the calculations have to be restricted until $\approx 10^{5} M_{\odot}$ in this study. Table 4 enlists a set of initial conditions for calculations that include the effect of residual gas expulsion, which will be discussed in Sect. 3. For now, we consider only the calculations in Table 3, which are for pure stellar clusters without any gaseous component.

Some of the computed models contain an initial primordial binary population (see Table 3 ). While including a population of primordial binaries makes the model more realistic, it also becomes much more computationally intensive. However, models including primordial binaries are essential to assess the role of the latter in expanding a young cluster. In the present computed models, we use a $f_{\mathrm{b}}(0)=100 \%$ primordial binary fraction that follow the "birth orbital period distribution". Such a primordial binary population gives rise to the appropriate period $(P)$ distribution observed for low-mass stellar binaries in the solar neighbourhood (Kroupa 1995). For massive stars of $m>5 M_{\odot}$, we adopt a much tighter and narrower initial period distribution 

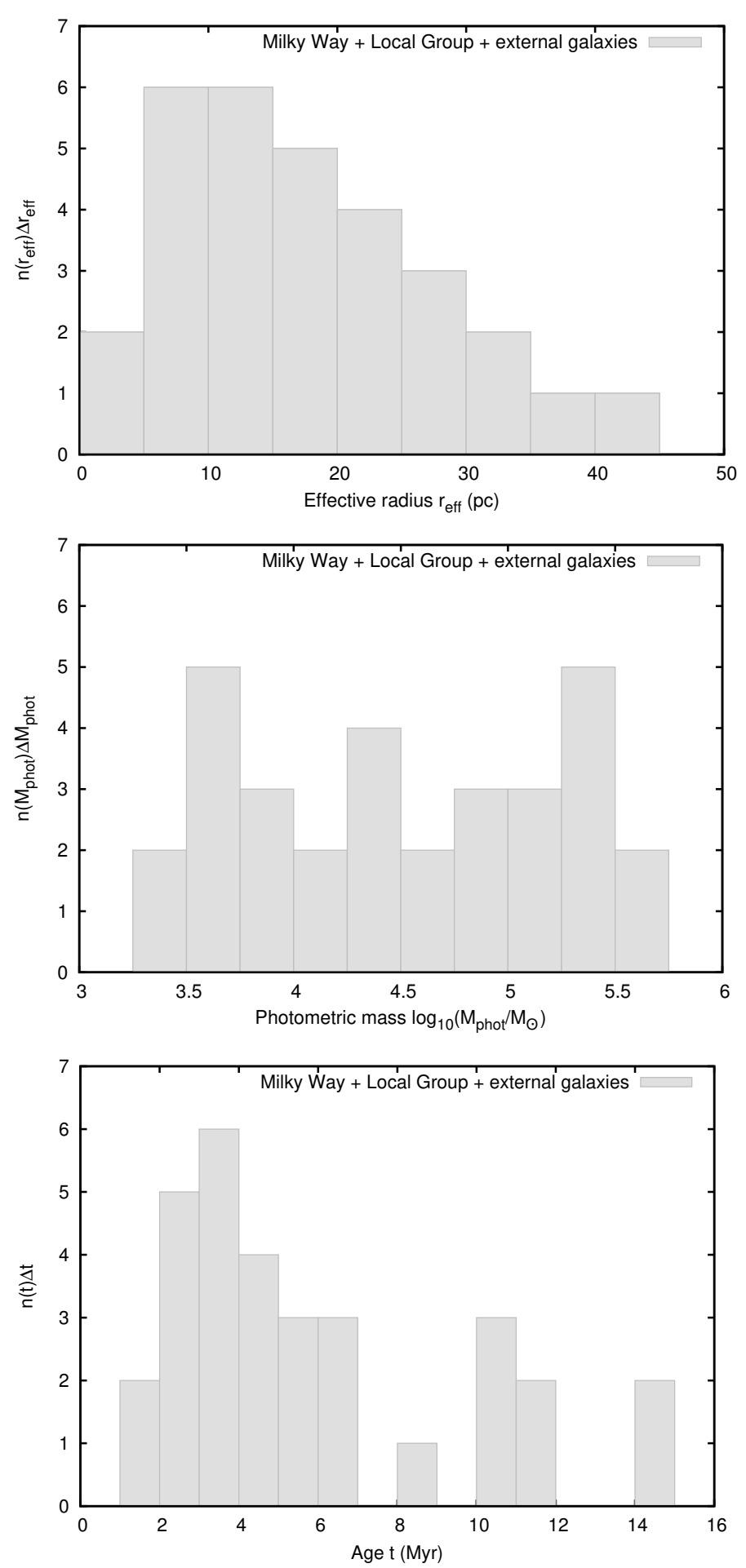

Fig. 2. Combined histograms of effecti ve radius $r_{\text {eff }}$ (top panel), photometric mass $M_{\text {phot }}$ (middle), and stellar age $t$ (bottom) for young massive stellar associations in the Milky Way, the Local Group, and in external galaxies. The data are from Table 2 .

given by a bi-modal Öpik law (uniform distribution in $\log _{10} P$ ) that spans over the range $0.3<\log _{10} P<3.5$, where $P$ is in days, as well as ordered pairing of binary components (i.e. binary mass ratio $\approx 1$ ). Both conditions are motivated by the population of O-star binaries, as observed in nearby massive clusters (Sana \& Evans 2011). Such observations also indicate $\gtrsim 70 \%$ binary fraction among O-star binaries, consistent with the binary fraction chosen in the present calculations. It is presently
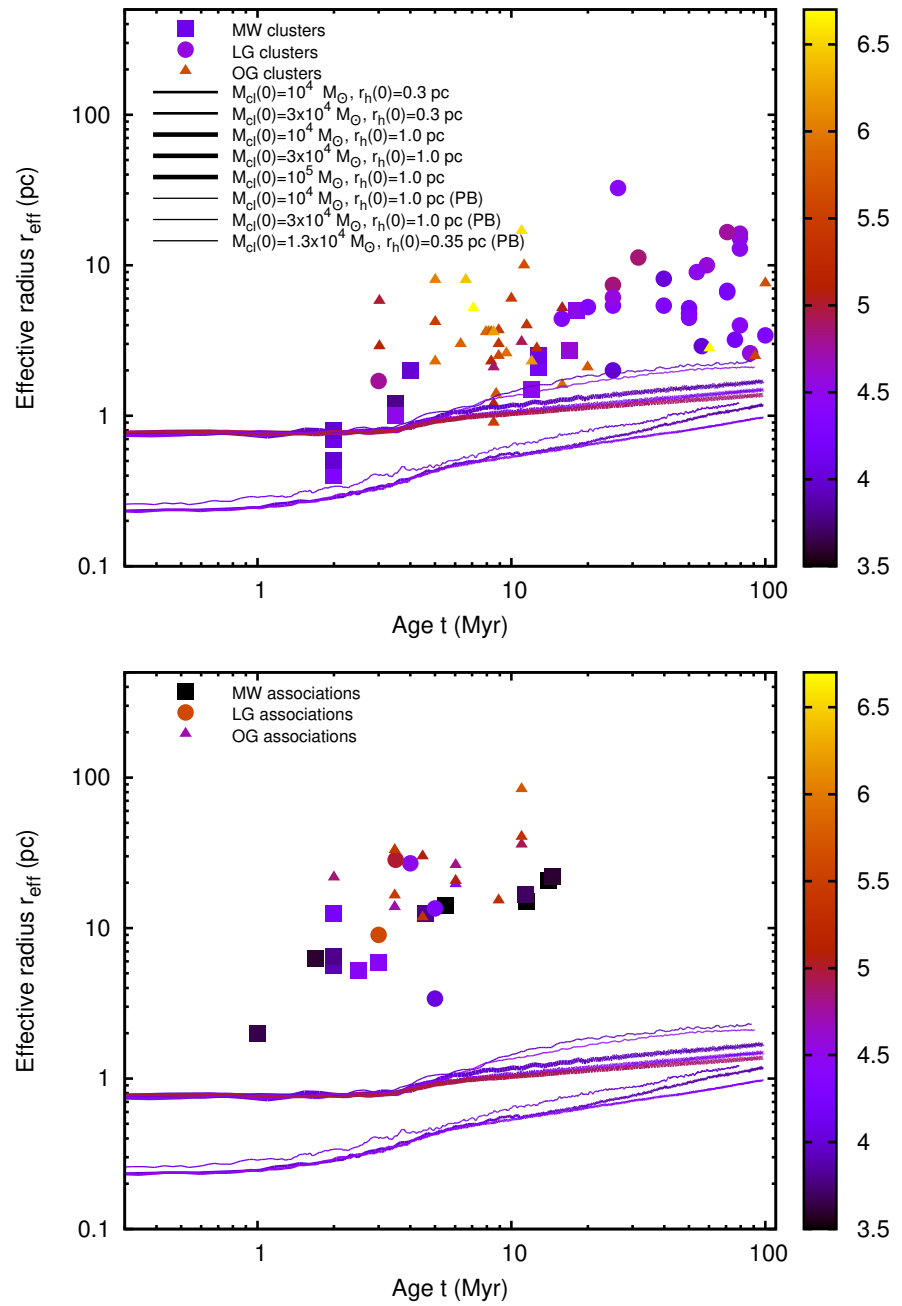

Fig. 3. Top: effective radius $r_{\text {eff }}$ vs. age $t$ for young massive bound star clusters (YMCs) in the Milky Way, the Local Group, and external galaxies which are distinguished by different filled symbols. The symbols are colour-coded according to the clusters' respective photometric mass, $\log _{10}\left(M_{\text {phot }} / M_{\odot}\right)$. These observed data are given in Table 1. Overlaid in the panel are the computed curves for the evolution of projected halfmass radius (or effective radius) $r_{\text {eff }}(t)$ for model star clusters with initial masses $M_{\mathrm{cl}}(0)$ and half-mass radii $r_{\mathrm{h}}(0)$ as given in Table 3 , which do not include a residual gas expulsion phase. These curves are distinguished according to the legends in the panel where "PB" indicates that the computed cluster includes a realistic primordial binary population (see Sect. 2.1). These lines are also colour-coded according to the corresponding clusters' instantaneous total bound mass $\log _{10}\left(M_{\mathrm{cl}}(t) / M_{\odot}\right)$. As can be seen, if the clusters evolve from compact sizes determined by substructures in molecular clouds, their secular expansion substantially falls short of the observed sizes of YMCs (see text). Bottom: here, the curves and the colour-coding are the same as above except that the data for the young massive associations in Table 2 are plotted.

unclear at which stellar mass and how the $P$-law changes and, therefore, in these computed models the discontinuous switching of the $P$-distribution at $m=5 M_{\odot}$ is chosen tacitly. Furthermore, primordial mass segregation is introduced in some of the initial models with binaries (see Table 3), using the method of Baumgardt et al. (2008). The mass segregation concentrates the massive, tight binaries in the cluster's center, maximizing the rate of dynamical encounters they undergo and hence their heating effect (see above). 
Table 3. Initial conditions for the computed model clusters without a gas expulsion phase (see Sect. 2.1).

\begin{tabular}{rccccccc}
\hline \hline$M_{\mathrm{cl}}(0) / M_{\odot}$ & $r_{\mathrm{h}}(0) / \mathrm{pc}$ & Primordial binaries & Stellar evolution & Mass segregation & $V_{*}(0) / \mathrm{km} \mathrm{s}^{-1}$ & $\tau_{\mathrm{cr}}(0) / \mathrm{Myr}$ & $\tau_{\mathrm{h}}(0) / \mathrm{Myr}$ \\
\hline $10^{4}$ & 0.3 & no & yes & no & 10.5 & 0.028 & 4.65 \\
$3 \times 10^{4}$ & 0.3 & no & yes & no & 18.1 & 0.017 & 8.05 \\
$10^{4}$ & 1.0 & no & yes & no & 5.8 & 0.172 & 28.30 \\
$3 \times 10^{4}$ & 1.0 & no & yes & no & 10.0 & 0.100 & 49.02 \\
$10^{5}$ & 1.0 & no & yes & no & 18.2 & 0.055 & 89.49 \\
$1.3 \times 10^{4}$ & 0.35 & yes & yes & yes & 11.7 & 0.030 & 6.68 \\
$10^{4}$ & 1.0 & yes & yes & yes & 6.0 & 0.167 & 28.30 \\
$3 \times 10^{4}$ & 1.0 & yes & yes & yes & 10.4 & 0.096 & 49.02 \\
\hline
\end{tabular}

Notes. The initial configurations are Plummer profiles with total mass, $M_{\mathrm{cl}}(0)$ and half-mass radius $r_{\mathrm{h}}(0)$. The corresponding values of the virial velocity dispersion (velocity dispersion within the virial radius $\approx r_{\mathrm{h}}(0)$ ), $V_{*}(0)$; the crossing time, $\tau_{\text {cr }}(0)=r_{\mathrm{h}}(0) / V_{*}(0)$; and the two-body relaxation time at half-mass radius, $\tau_{\mathrm{h}}(0)$, are given. Also indicated is whether the initial system includes primordial binaries (see Sect. 2.1) and whether it is mass segregated (as in Baumgardt et al. 2008). Stellar evolution (see Sect. 2.1) is used in all $N$-body calculations. The initial clusters are always located at $R_{\mathrm{G}} \approx 8 \mathrm{kpc}$ Galactocentric distance (the solar distance) and they orbit the Galactic center with $V_{\mathrm{G}} \approx 220 \mathrm{~km} \mathrm{~s}^{-1}$.

Table 4. Initial conditions and gas expulsion parameters for the computed model clusters that include a gas expulsion phase.

\begin{tabular}{rccccccc}
\hline \hline$M_{\mathrm{cl}}(0) / M_{\odot}$ & $r_{\mathrm{h}}(0) / \mathrm{pc}$ & $M_{\mathrm{g}}(0) / M_{\odot}$ & $V_{*}(0) / \mathrm{km} \mathrm{s}^{-1}$ & $V_{*}^{\prime}(0) / \mathrm{km} \mathrm{s}^{-1}$ & $\tau_{\mathrm{cr}}^{\prime}(0) / \mathrm{Myr}$ & $\tau_{\mathrm{g}} / \mathrm{Myr}$ & $\tau_{\mathrm{d}} / \mathrm{Myr}$ \\
\hline $10^{4}$ & 0.15 & $2 \times 10^{4}$ & 14.7 & 25.5 & 0.006 & 0.015 & 0.6 \\
$3 \times 10^{4}$ & 0.15 & $6 \times 10^{4}$ & 25.8 & 44.7 & 0.003 & 0.015 & 0.6 \\
$10^{4}$ & 0.30 & $2 \times 10^{4}$ & 10.5 & 18.2 & 0.016 & 0.030 & 0.6 \\
$3 \times 10^{4}$ & 0.30 & $6 \times 10^{4}$ & 18.1 & 31.3 & 0.010 & 0.030 & 0.6 \\
$10^{5}$ & 0.30 & $2 \times 10^{5}$ & 33.1 & 57.3 & 0.005 & 0.030 & 0.6 \\
$10^{4}$ & 1.0 & $2 \times 10^{4}$ & 5.8 & 10.0 & 0.100 & 0.100 & 0.6 \\
$3 \times 10^{4}$ & 1.0 & $6 \times 10^{4}$ & 10.0 & 17.3 & 0.058 & 0.100 & 0.6 \\
$10^{5}$ & 1.0 & $2 \times 10^{5}$ & 18.2 & 31.5 & 0.032 & 0.100 & 0.6 \\
\hline $3 \times 10^{4}$ & 1.0 & $6 \times 10^{4}$ & 9.9 & 17.1 & 0.058 & $2.5,5.0$ & 0.0 \\
$3 \times 10^{4}$ & 2.0 & $6 \times 10^{4}$ & 7.0 & 12.1 & 0.165 & $2.5,5.0$ & 0.0 \\
$3 \times 10^{4}$ & 3.0 & $6 \times 10^{4}$ & 5.8 & 10.0 & 0.300 & $2.5,5.0$ & 0.0 \\
$3 \times 10^{4}$ & 4.0 & $6 \times 10^{4}$ & 5.0 & 8.7 & 0.460 & $2.5,5.0$ & 0.0 \\
$10^{5}$ & 2.0 & $2 \times 10^{5}$ & 12.9 & 22.3 & 0.090 & 5.0 & 0.0 \\
$10^{5}$ & 3.0 & $2 \times 10^{5}$ & 10.5 & 18.2 & 0.165 & 5.0 & 0.0 \\
$10^{5}$ & 4.0 & $2 \times 10^{5}$ & 9.1 & 15.8 & 0.253 & 5.0 & 0.0 \\
\hline
\end{tabular}

Notes. The columns from left to right give (a) total initial stellar mass $M_{\mathrm{cl}}(0)$; (b) initial stellar half-mass radius $r_{\mathrm{h}}(0)$; (c) initial gas (potential, see Sect. 3) mass $M_{\mathrm{g}}(0)$; which follows the stellar profile, (d) virial velocity dispersion $V_{*}(0)$; (e) scaled velocity dispersion $V_{*}^{\prime}(0)$; (f) the corresponding crossing time (dynamical time) $\tau_{\mathrm{cr}}^{\prime}(0)$; (g) timescale for gas (potential) dilution $\tau_{\mathrm{g}}$; and (h) the time $\tau_{\mathrm{d}}$, at which the gas expulsion begins. Here, $V_{*}^{\prime}(0)$ is the virial velocity dispersion at which the stellar velocities are boosted so that the intended $r_{\mathrm{h}}(0)$ can be maintained when the gas potential is applied. For $M_{\mathrm{g}}(0) \approx 2 M_{\mathrm{cl}}(0)$ (i.e. $\epsilon \approx 33 \%$ star formation efficiency), as it is here, $V_{*}^{\prime}(0) \approx \sqrt{3} V_{*}(0)$, which determines the dynamical time, $\tau_{\mathrm{cr}}^{\prime}(0)$, of the initial gas-embedded clusters. These initial clusters are located at $R_{\mathrm{G}} \approx 8 \mathrm{kpc}$ Galactocentric distance (the solar distance) and they orbit the Galactic center with $V_{\mathrm{G}} \approx 220 \mathrm{~km} \mathrm{~s}^{-1}$.

\subsection{Secular expansion}

The solid curves in the panels of Fig. 3 show the computed evolution of effective radius, $r_{\text {eff }}(t)$, for the models in Table 3. The same set of curves are used for both upper and lower panels which contain the data points (filled symbols) for observed YMCs and associations (see Sect. 1), respectively. Here the instantaneous $r_{\text {eff }}(t)$ is obtained by taking the arithmetic mean of the projected half-mass radii $(50 \%$ Lagrange radius integrated over a plane) over three mutually perpendicular planes passing through the cluster's density center. It can be seen that beginning from sizes similar to that of the substructures in molecular clouds (see Sect. 1), it is impossible to attain the observed sizes of YMCs and associations in 100 Myr. We note that realistic conditions are used in these computed models which include stellar mass loss, retention of $\approx 50 \%$ of the BHs and NSs formed via supernovae, and include a realistic population of tight massive primordial binaries (see Sect. 2.1). For test purposes, a few 


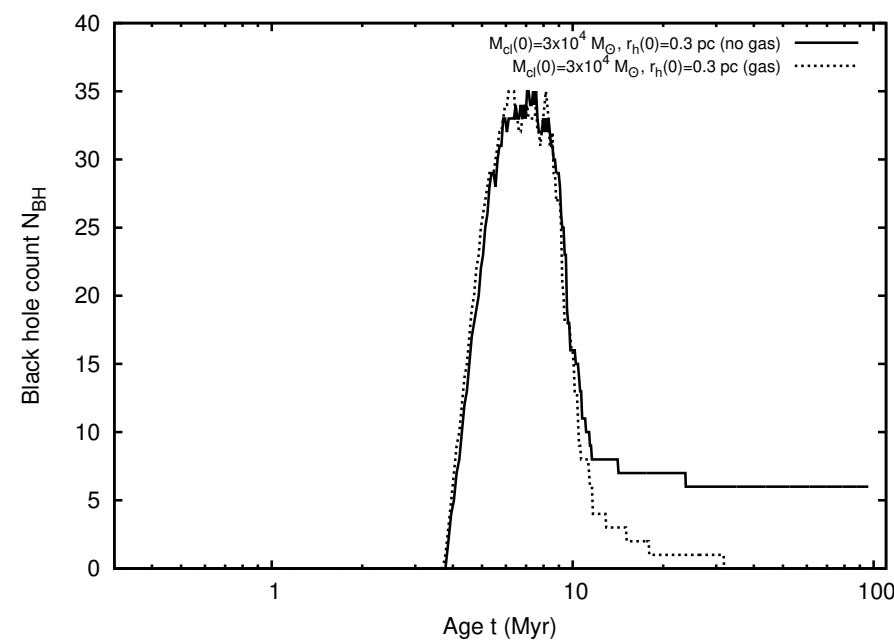

Fig. 4. Evolution of the population $N_{\mathrm{BH}}$ of stellar-mass black holes (BHs) bound within the computed clusters. The total number of BHs formed by supernovae is $\approx 80$ in each case, about half of which are retained in the cluster following their birth (by applying low natal kicks). See text for additional details.

computed models begin with a significantly more extended size, namely, $r_{\mathrm{h}}(0) \approx 1.0 \mathrm{pc}$, which can nearly reach the sizes of the most compact observed YMCs, but are still much more compact than most YMCs and associations. In all calculations, the cluster expands much more quickly than its initial half-mass (two-body) relaxation time, $\tau_{\mathrm{h}}(0)$ (cf. Table 3 ), since the initial expansion is driven by stellar-evolutionary mass loss (see above) that happens on a much shorter timescale.

Notably, starting from the same $r_{\mathrm{h}}(0)$ and for evolution time $t \lesssim 10 \mathrm{Myr}$, the computed growth of $r_{\text {eff }}(t)$ is nearly independent of the initial cluster mass $M_{\mathrm{cl}}(0)$. As can be expected, the latetime growth of $r_{\text {eff }}$ is somewhat larger for the models including primordial binaries (Sect. 2.1). The clusters also retain BHs and NSs that aid late-time expansion. Figure 4 (solid curve) shows the time evolution of the number of $\mathrm{BHs}\left(N_{\mathrm{BH}}\right)$ bound to the cluster in one of the calculations (see also Sect. 3.1). However, the computed values of $r_{\text {eff }}$ always fall substantially short of the observed values for YMCs and associations.

We note that the computed expansion of the above model clusters are "natural" in the sense that they are driven purely by dynamics and mass loss of the constituent stars. In other words, the expansion is due to physical processes that would co-exist in any star cluster that begins its life with a realistic zero-age stellar population. Given that the dynamics part in the NBODY7 calculation is accurate owing to star-by-star $N$-body integration avoiding force softening, the primary uncertainties can arise from stellar evolution mass loss, in which analytic recipes are used (see Sect. 2.1). However, stellar evolution primarily influences the cluster expansion during supernovae when the majority of the mass of a massive star (ZAMS mass $>8 M_{\odot}$ ) is lost essentially instantaneously leaving behind an NS (typically 2-3 $M_{\odot}$ ) or a $\mathrm{BH}\left(10-30 M_{\odot}\right.$ for solar metallicity). The supernova ejecta typically exceed $10^{4} \mathrm{~km} \mathrm{~s}^{-1}$ and would escape any star cluster. Hence the dominant mass loss during the supernovae phase $(\approx 3.5-50 \mathrm{Myr}$ ) effectively grosses over the uncertain details of stellar winds during main sequence and late evolutionary stages. There are also uncertainties introduced from the treatments of binary evolution undergoing mass transfer, stellar collisions, and binary mergers, but these have much less impact on a cluster's expansion than supernovae.

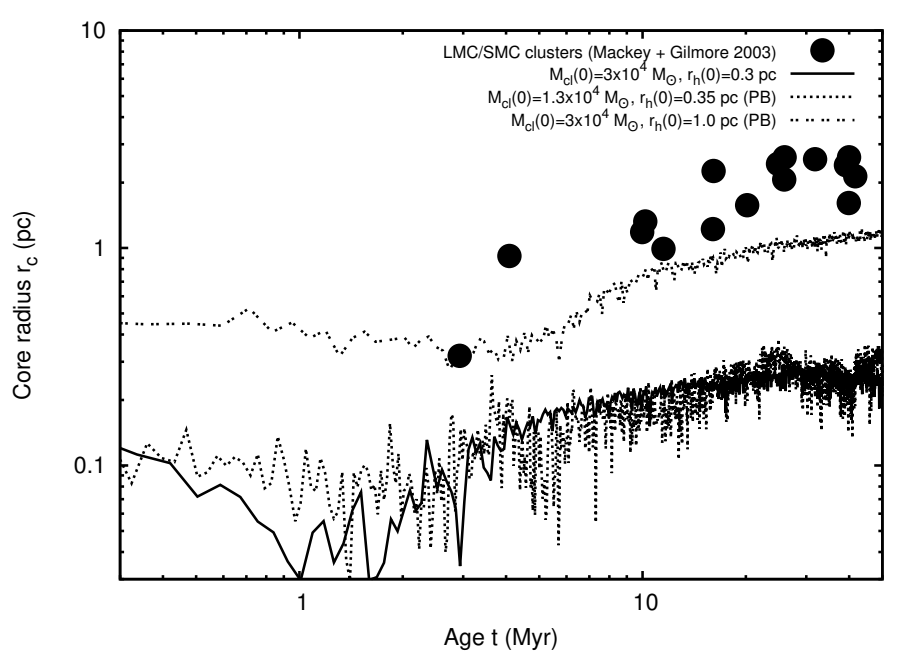

Fig. 5. Evolution of core radii (solid and dashed curves) $r_{\mathrm{c}}$ for representative computed clusters without a gas expulsion phase ("PB" indicates inclusion of primordial binaries). Like the half-mass radii, the computed values of $r_{\mathrm{c}}$, starting from the filament-like compact initial conditions (see above), substantially fall short of the observed core radii of LMC and SMC clusters (filled circles). The latter data are obtained from Mackey \& Gilmore (2003a,b); here, the relevant data are extracted from Fig. 1 of Mackey et al. (2008) using the standalone DEXTER service (http://dc . zah. uni-heidelberg.de/sdexter).

In the present calculations, all of the stellar mass loss (wind or supernova ejecta) are removed from the system. In reality, a fraction of the stellar wind can be slower than the cluster's escape speed and retain in the cluster. This is also true for the material ejected during massive stellar collisions and massive binary mergers (de Mink et al. 2009). Furthermore, gas can be externally accreted if the cluster happens to interact with a nearby molecular cloud (Pflamm-Altenburg \& Kroupa 2009). All of this material is either generated in situ (e.g. for massive stellar winds and massive binary ejecta) or eventually collected at the cluster's center as a cooling flow. As often argued, such a gas reservoir in a star cluster can result in the formation of second-generation stars (Gratton et al. 2012). A quantification of this effect is beyond the scope of this paper. However, the central gas collection can only counteract the effect of gravitational potential dilution from stellar mass loss, inhibiting the cluster's expansion until the first supernova. During the supernovae, energy injected into any residual gas is sufficient to eliminate it from the cluster.

In other words, while the details of stellar winds from massive stars is still ambiguous, it is the supernovae that ultimately determines the impact of the stellar mass loss on a cluster's expansion during its first $\approx 50 \mathrm{Myr}$. The details of the Type-II supernova mechanism (which is largely under debate) is also irrelevant here; the key quantity is the total mass loss, which is determined by the remnant masses. The above mass ranges of NSs and BHs, which are adopted in NBODY7, are supported by observations in regions with solar-like metallicity. For lower metallicities, e.g. in the Magellanic Clouds and several other Local Group dwarf galaxies, the BHs are heavier and the overall stellar mass loss is smaller. After the supernovae phase, the primary ambiguous factor that contributes to the cluster's expansion is the population of BHs and NSs retained at birth, which are taken to be substantial $(\approx 50 \%)$ in the present computations.

Figure 5 shows the evolution of core radii, $r_{\mathrm{c}}(t)$, for representative computed models from Table 3 . The data points in this figure are the observed core radii of LMC and SMC clusters from Mackey \& Gilmore (2003a,b). The computed $r_{\mathrm{c}}(t) \mathrm{s}$ 
corresponding to the compact profiles again fall far short of the observed values. In this context, it is important to note that Mackey et al. (2008) also computed the $r_{\mathrm{c}}$ evolution for model clusters using direct $N$-body calculations, which agreed reasonably with the above data. These authors used these computations to demonstrate the dynamical heating effect of stellar mass BHs (see above) and its effect on cluster size. However, unlike the present calculations, those of Mackey et al. (2008) start with wider $r_{\mathrm{h}}(0) \gtrsim 1 \mathrm{pc}$ clusters, all the BHs are retained after formation, and the $\mathrm{BHs}$ are generally more massive since lower $\left(0.5 Z_{\odot}\right)$ LMC metallicity is used. Also, unlike here, ElsonFall-Freeman (EFF) profiles are used as initial configurations. These points explain the differences between the present and the Mackey et al. (2008) computations. Notably, unlike $r_{\mathrm{c}}, r_{\mathrm{eff}}$ approximates the virial radius of a cluster in (or near) dynamical equilibrium and hence is nearly independent of the cluster's density profile.

The above discussion implies that although the present evolutionary calculations include less understood physical phenomena such as massive stellar evolution, core-collapse supernova, and stellar mergers, it is unlikely that a truly realistic treatment of these factors would cause the clusters to expand at a much higher rate than the presently computed ones and reach the observed sizes. This calls for additional mechanisms that would be necessary for a newly assembled compact cluster to reach the present-day observed sizes.

\section{Non-secular evolution of star clusters: primordial gas expulsion}

The computations in Sect. 2.2 do not assume any primordial gas present initially in the cluster, i.e. the cluster is taken to be formed with $100 \%$ local SFE. A high local SFE has been claimed by several authors based on hydrodynamic calculations without feedback processes or with partially implemented feedback processes (e.g. Klessen et al. 1998; Bate \& Bonnell 2004; Kruijssen et al. 2012; Girichidis et al. 2012; Dale et al. 2015). Clearly, as the computations in Sect. 2.2 show, if a young cluster is hatched along a molecular-gas filament or at a filament junction with effectively $100 \%$ SFE, it cannot evolve to the presently observed sizes of YMCs and associations; their effective radii would fall short by 1-2 orders of magnitude even after $100 \mathrm{Myr}$ of evolution. There are two possible ways more extended clusters could form, viz., (a) residual gas dispersal and (b) mergers of less massive, compact sub-clusters.

Observations of molecular clouds and embedded clusters suggest that in regions of high star-formation activity, the local SFE typically varies between a few percent to $\approx 30 \%$. The SFE of embedded clusters in the solar neighbourhood can also be at most $\approx 30 \%$ (Lada \& Lada 2003). This is supported by high-resolution radiation magneto-hydrodynamic (MHD) simulations of proto-star formation (Machida \& Matsumoto 2012; Bate et al.2014). Although such computationally expensive simulations are limited to the spatial scale of a proto-star, they suggest a maximum $\approx 30 \%$ SFE for proto-star formation, consistent with observations. This limit occurs because of the interplay between gas accretion onto the proto-star (sink particle) and radiatively and magnetically driven outflows from the proto-star (Bate et al. 2014), introducing self-regulation in the process. This is also supported by the profusion of outflow activities observed from high-mass and low-mass proto-stars (e.g. Bally et al. 2012, 2015). The above would then imply that the maximum SFE over a region forming a population of proto-stars is also $30 \%$ or less. Hence, it is realistic to assume that a protocluster is initially embedded in a substantial primordial gas, the latter being subsequently cleared from the system by the stellar feedbacks (e.g. radiation, outflows).

A widely studied scenario that efficiently expands a cluster from its compact initial conditions is the "explosive" expulsion of the residual gas (i.e. on a timescale comparable to the cluster's dynamical time). In this study, we utilize the widely-used method of treating the background residual gas as an external spherically symmetric potential, co-centric with the cluster. The effect of gas dispersal is mimicked by depleting this potential exponentially, which captures the overall dynamical effect of the removal of gas (due to radiative and mechanical feedback from the ionising O/B stars; see Banerjee \& Kroupa 2013, 2015b, for an overview). The effect of gas dispersal on the stellar cluster depends on three primary parameters, viz., the SFE, $\epsilon$; the timescale, $\tau_{\mathrm{g}}$, or the effective velocity, $v_{\mathrm{g}}\left(\tau_{\mathrm{g}}=r_{\mathrm{h}}(0) / v_{\mathrm{g}}\right)$, of expelling gas; and the time of the commencement of the gas dispersal, $\tau_{\mathrm{d}}$. In the present calculations, we take the representative values $\epsilon \approx 33 \%, v_{\mathrm{g}} \approx 10 \mathrm{~km} \mathrm{~s}^{-1}$ (the sound speed in ionized hydrogen), $\tau_{\mathrm{g}} \approx 0.6 \mathrm{Myr}$; see Banerjee \& Kroupa (2013) for details. However, we also consider the possibility of a slower rate of gas expulsion (see Sect. 3.2). The relative density distribution of the stars and gas is also crucial for the impact of gas expulsion (Boily \& Kroupa 2003a,b). Here we take both the stellar and gas distribution to follow the same Plummer distribution, i.e. the residual gas and the stars are ideally coupled without radial variation of $\epsilon$, maximizing the effect of gas expulsion (see also Sect. 5).

\subsection{Calculations involving explosive gas expulsion}

The first part of Table 4 lists the initial conditions for the computed models containing residual gas which is expelled at the HII sound speed, i.e. with $v_{\mathrm{g}} \approx 10 \mathrm{~km} \mathrm{~s}^{-1}$. The corresponding potential depletion timescales, $\tau_{\mathrm{g}}$, are comparable with the clusters' initial crossing (dynamical) times, $\tau_{\text {cr }}^{\prime}(0)$; see Table 4 (first part). This makes the gas expulsion explosive, i.e. the stellar system fails to relax (via two-body interactions) and adjust with the depleting potential, thereby beginning to expand with its own dynamical time.

Figure 6 shows the $r_{\text {eff }}$ evolutions of the above models which are compared to the observed $r_{\text {eff }}$ values of YMCs and associations (upper and lower panels, respectively). With an explosive gas expulsion of about $70 \%$ by mass, the filament-like compact clusters can expand to reach the observed sizes of most of the MW YMCs (Fig. 6, upper panel). On the other hand, most of the LG and OG YMCs and the associations are still a few factors larger in size than the computed models with compact initial conditions $\left(r_{\mathrm{h}}(0) \lesssim 0.3 \mathrm{pc}\right)$. Unless these wider YMCs and associations are closely packed ensembles of more compact and less massive (sub-)clusters, the corresponding $r_{\text {eff }}$ values can only be arrived at from more diffuse initial configurations with $r_{\mathrm{h}}(0) \approx 1 \mathrm{pc}$, as seen in Fig. 6 . On the other hand, $r_{\mathrm{h}}(0) \approx 1 \mathrm{pc}$ clusters lose more stars and become lighter in their expanded phase than their more compact counterparts (see colour-coding of the curves in Fig. 6).

Figure 7 shows the evolution of the bound mass, $M_{\mathrm{cl}}(t)$, for the computed models in Table 4 (first part), which are compared with the photometric masses, $M_{\text {phot }}$, of the observed YMCs and associations. It shows that so far only the clusters' masses are concerned, the computed initial mass range of $10^{4} M_{\odot}<$ $M_{\mathrm{cl}}(0)<10^{5} M_{\odot}$ reasonably covers the $M_{\text {phot }}-t$ space of the MW and LG YMCs and associations. The OG sample comprises 

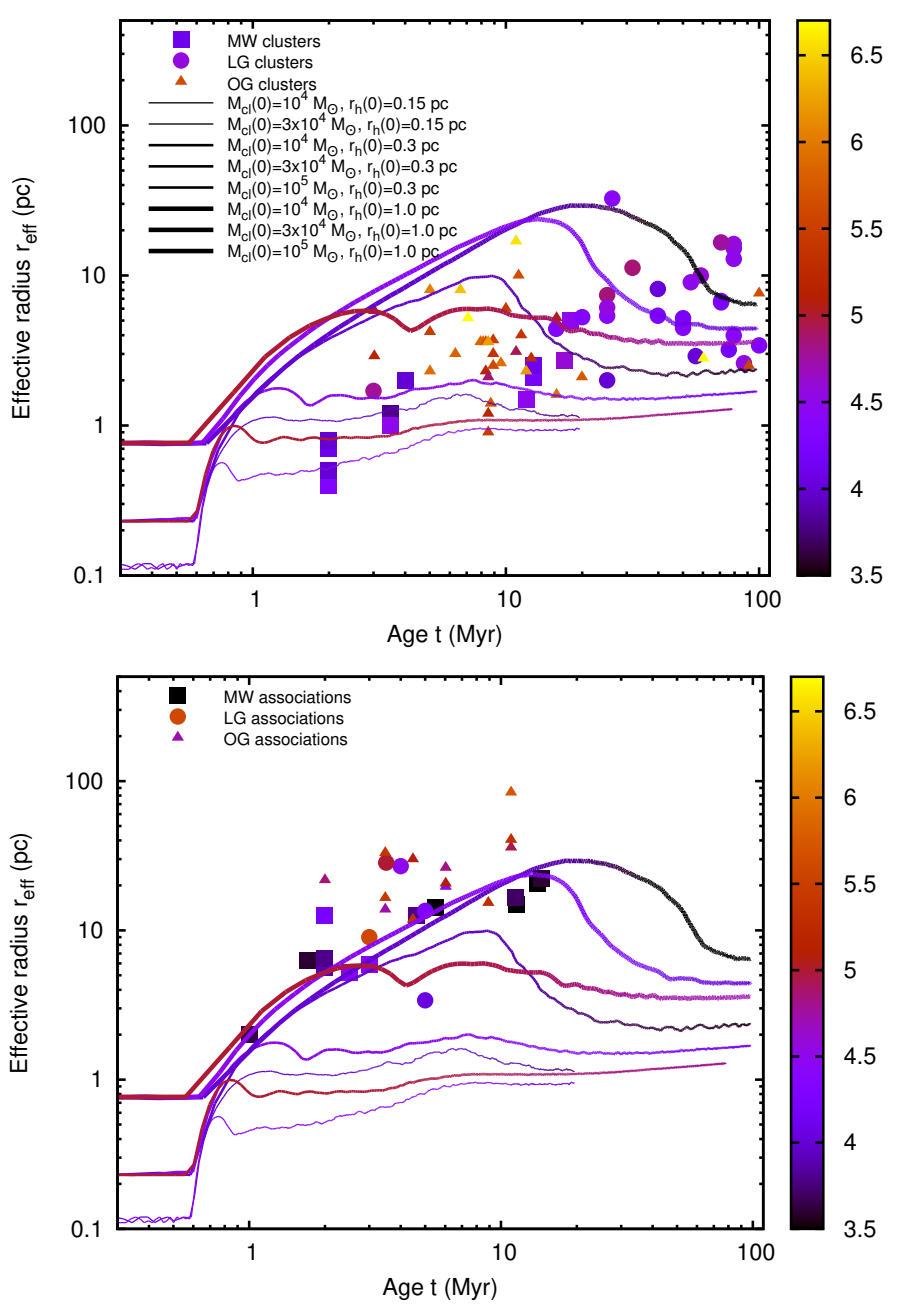

Fig. 6. Data points and their colour-coding in both panels are identical to Fig. 3. The curves are the computed evolution of the projected half-mass radii (or effective radii) $r_{\text {eff }}(t$ ), including a gas dispersal phase with star formation efficiency $\epsilon \approx 33 \%$ (see Table 4, top part and text for details). As in Fig. 3, the curves also follow the same colour-coding according to the corresponding clusters' instantaneous total bound mass $\log _{10}\left(M_{\mathrm{cl}}(t)\right)$ (the same set of curves are overlaid on both panels). These figures imply that even if the YMCs evolve from filament-like compact sizes, such substantial (and explosive) gas dispersal will expand them to their present observed sizes (half-mass radii) in the Milky way and in the Local Group (top panel). However, to reach the sizes of the most extended Local Group YMCs, they need to evolve from $r_{\mathrm{h}}(0) \gtrsim 1 \mathrm{pc}$ half-mass radii, unless such objects are low-mass cluster complexes (Brüns et al. 2009). The latter is also true for the young massive associations (bottom panel). See text for details.

10-100 times more massive systems (see Sect. 1) and hence similarly larger values of $M_{\mathrm{cl}}(0)$ are required to cover these masses, which is beyond the present scope (see Sect. 2.1). However, it can be said that such massive clusters would lose much less mass than their less massive (computed) counterparts (see below).

From Fig. 6, a general trend can be recovered: beginning from a given $r_{\mathrm{h}}(0)$ and for fixed $\epsilon$ and $v_{\mathrm{g}}$, a cluster with larger $M_{\mathrm{cl}}(0)$ loses less mass and also expands less. This trend is what can be expected since for a fixed $r_{\mathrm{h}}(0)$, larger $M_{\mathrm{cl}}(0)$ implies a larger binding energy for the system. Also, their higher stellar density results in more efficient violent relaxation causing a larger fraction of stars to be retained during the expansion due to gas expulsion. This trend, however, makes it difficult to achieve the observed $r_{\mathrm{eff}} \mathrm{s}$ of most of the extended YMCs $\left(r_{\mathrm{eff}} \gtrsim 1 \mathrm{pc}\right)$
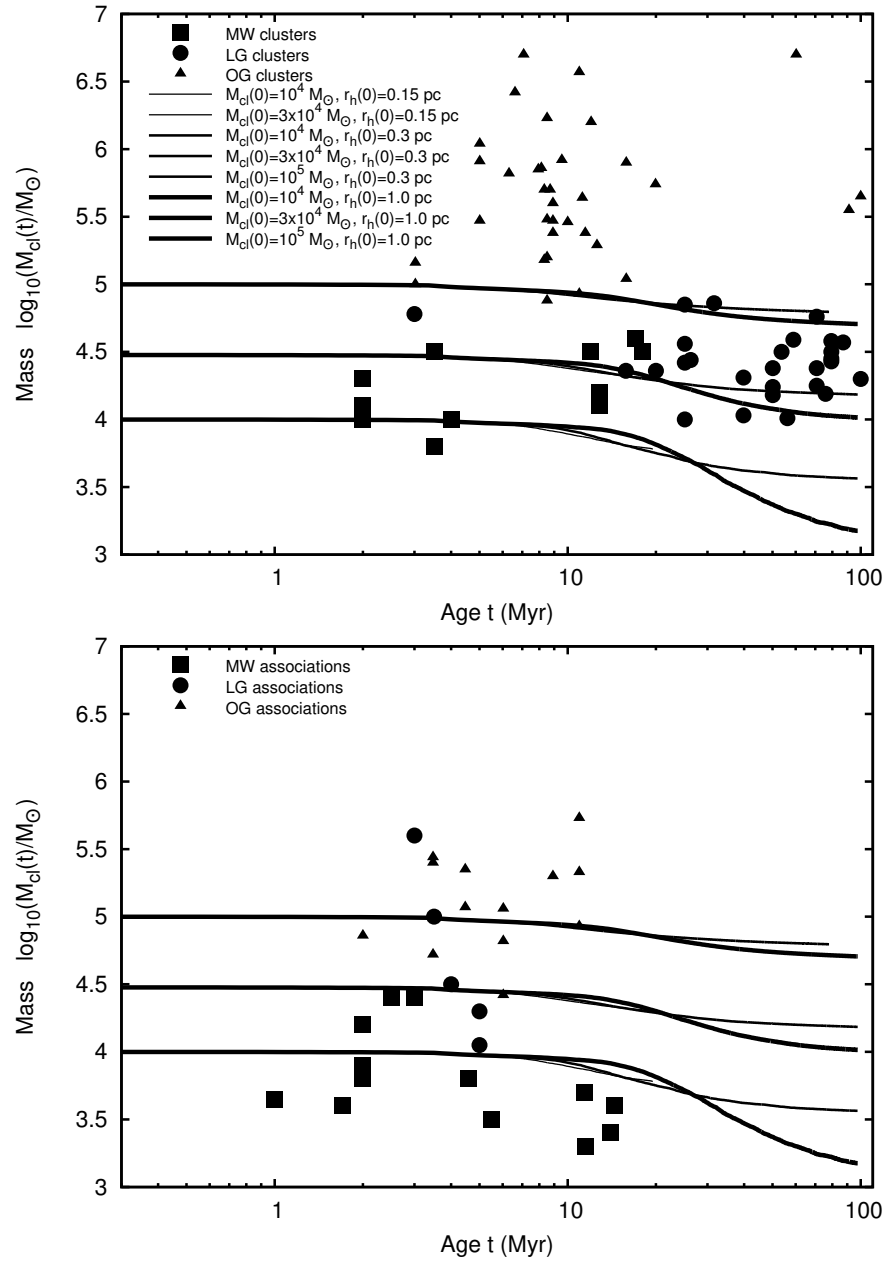

Fig. 7. Curves give the evolution of the instantaneous total bound stellar mass $\log _{10}\left(M_{\mathrm{cl}}(t)\right)$ for the calculations in Fig. 6 that include an initial gas dispersal phase (see Table 4, top part and text). In both panels, data points corresponding to the same young massive clusters and associations (in the top and bottom panels, respectively) as in Figs. 3 and 6 are used, but without colour-coding. The same set of curves are overlaid on both panels and the legends are given in the top panel.

starting from the compact initial conditions without compromising the mass, as Fig. 7 shows.

Starting from $r_{\mathrm{h}}(0)=0.3 \mathrm{pc}$, the computed cluster with $M_{\mathrm{cl}}(0) \approx 10^{4} M_{\odot}$ reaches the sizes of most MW YMCs while still more compact than most of the LG VYMCs, but its mass falls far short of the VYMC masses (cf. Fig. 7). The more massive $r_{\mathrm{h}}(0)=0.3 \mathrm{pc}$ clusters maintain VYMC-like masses, but fall far short of the observed sizes of most VYMCs. While the OG clusters exceed the computed mass range, it is clear that the size discrepancy would only increase for them. On the other hand, with the $r_{\mathrm{h}}(0) \approx 1 \mathrm{pc}$ models only the largest $r_{\mathrm{eff}} \mathrm{s}$ are reachable, but again compromising $M_{\mathrm{cl}}$ (see Figs. 6 and 7). The same holds if the SFE is decreased instead. The above discussion implies that while the most compact MW, LG (and OG) YMCs are naturally achievable from compact filament-sized initial clusters via residual gas expulsion in terms of their masses and sizes, the same clusters seem to require fine tuning for the more extended VYMCs (and the associations).

Finally, Fig. 4 shows the evolution of $N_{\mathrm{BH}}$ for a computed model with explosive gas expulsion (dotted curve). The lesser retention of $\mathrm{BHs,}$ in this case, is likely due to larger expansion and hence lower central density of the cluster. The lower 
central stellar density largely reduces the dynamical friction on the mass-segregated BHs comprising the central $\mathrm{BH}$ subcluster (also known as the "black core"; Mackey et al. 2008). This causes them to effectively act like an isolated small- $N$ cluster, having short two-body relaxation time, hence they evaporate rapidly. The effect of a star cluster's birth condition on its compact-star population is itself an emerging topic with wide implications, which will be presented elsewhere.

\subsection{Placid or slow gas dispersal and its implications}

Contrary to (near) explosive gas expulsion, a "placid" mode of gas expulsion similarly expands a cluster, but causes much less mass loss in stars. In this case, the gas is dispersed over a timescale that is much longer than the cluster's crossing (dynamical) time. This allows the cluster to adjust itself with the changing potential and expand while remaining in near dynamical equilibrium, i.e. it can evolve in a quasi-static (or adiabatic; Kroupa 2008) fashion. The timescale of expansion is, of course, determined by the gas dispersal time $\tau_{\mathrm{g}} \gg \tau_{\mathrm{cr}}(0)$. The cluster, therefore, expands much more slowly than the abrupt expansion at $\tau_{\mathrm{d}}$ for the explosive case (see Sect. 3.1).

A motivation for considering the possibility of such slow gas dispersal is several observed gas-embedded regions containing massive O/B-stars that are several Myr old. Perhaps the most well-studied example is the Galactic embedded region W3 Main that has been continuously forming stars for at least $3 \mathrm{Myr}$ (Feigelson \& Townsley 2008; Bik et al. 2014). Another more recently cited example is the W33 complex (Messineo et al. 2015). In such regions, while there are individual ionized bubbles around each massive star, the overall stellar system still seems to be deeply embedded in molecular gas. A common feature of such embedded stellar populations is that the stars are distributed in a substructured fashion over significantly larger spatial scales than (near) spherical clusters; typically over a few parsecs to 10 s of parsecs. While, at present, it is unclear why such systems fail to clear the gas early, unlike the starburst clusters, the wider distribution of the ionizing $\mathrm{O} / \mathrm{B}$ stars can be a clue. As argued in Banerjee \& Kroupa (2015b), such a condition might lead to evacuation of gas only around individual OB stars and not in bulk. In this case, the overall gas is dispersed in a much longer timescale than dictated by $v_{\mathrm{g}} \approx 10 \mathrm{~km} \mathrm{~s}^{-1}$, which is also much longer compared to the stellar system's dynamical time (i.e. placidly).

The second part of Table 4 lists the initial conditions for computed models where longer $\tau_{\mathrm{g}}=2.5 \mathrm{Myr}$ and $5 \mathrm{Myr}$ are considered. The values of $M_{\mathrm{cl}}(0)$ are similar to the previously computed models, but much wider $1.0 \mathrm{pc}<r_{\mathrm{h}}(0)<4.0 \mathrm{pc}$ are considered. For simplicity, we consider only initially spherical systems without any substructures since the objective is to study the overall mass and size evolution of the system. Figure 8 shows the corresponding computed $r_{\text {eff }}(t)$ s. A few additional computed models with $v_{\mathrm{g}} \approx 10 \mathrm{~km} \mathrm{~s}^{-1}$ are shown for comparison. As expected, $M_{\mathrm{cl}}$ does not decline significantly during the evolution. Most of the stellar mass loss is due to the supernovae ejecta and dynamically ejected stars which is much lower than that due to stars becoming unbound by explosive gas expulsion. Interestingly, unlike the case of explosive expulsion, placid expulsion causes clusters of different $M_{\mathrm{cl}}(0)$ to approach nearly the same $r_{\mathrm{eff}}$ for a given $r_{\mathrm{h}}(0)$ (and $\epsilon$ ); because the associated stellar mass loss being small compared to the total bound mass, the final cluster size is essentially determined by the relative depletion of the background gas potential. This is as expected from adiabatic cluster evolution (Kroupa 2008). Figure 9 compares the evolution of the

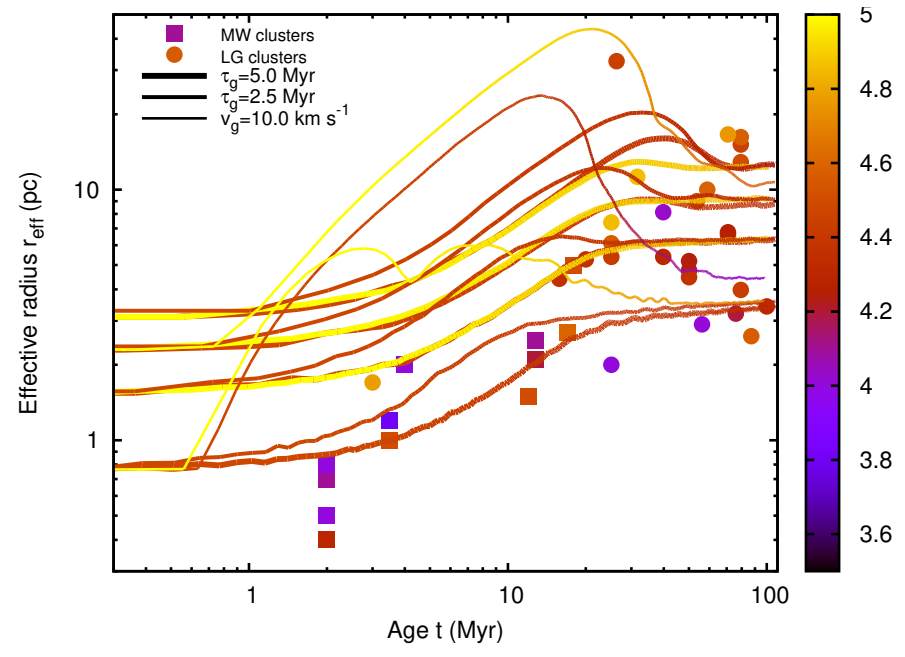

Fig. 8. Curves representing the evolution of projected half-mass radii (or effective radii) $r_{\text {eff }}(t)$ for computed model clusters that include a gas expulsion phase. Unlike those in the previous figures, these computed clusters initiate from much more extended stellar distributions with initial half-mass radius, $r_{\mathrm{h}}(0)>1 \mathrm{pc}$ (see Table 4 , bottom). Furthermore, the gas expulsion timescales, $\tau_{\mathrm{g}}$, are much longer as indicated in the legend, making the gas expulsion placid in these cases (except for $v_{\mathrm{g}}=10 \mathrm{~km} \mathrm{~s}^{-1}$, which causes explosive expulsion as in the previous cases). For $\epsilon \approx 30 \%$ star formation efficiency, these extended model clusters expand to the observed sizes of the most extended young massive clusters in the Local Group (filled symbols), while also covering the mass ranges appropriate for them. We note that with slow or placid gas expulsion, the clusters tend to reach sizes that are nearly independent of their initial masses, unlike their explosive counterparts. As for the previous figures, the symbols and the curves are colour-coded according to the corresponding current total (photometric) mass.

virial ratio, $Q$, between different modes of cluster expansion; see caption for explanation. The above property implies that in order to achieve an observed cluster size through slow gas expulsion, only the appropriate initial size needs to be set, i.e. fine tuning is hardly necessary.

The main drawback of explaining the extended YMCs (or any cluster in general) as an outcome of slow gas dispersal is that the depletion process is unlikely to remain slow (or placid) for its entire duration. When the Type-II supernovae commence from $\approx 4$ Myr age, their energy input into the gas could be sufficient to eject the gas explosively. If this happens, then, for the extended initial clusters used in Fig. 8, the rapid gas expulsion (together with the tidal field) would largely dissolve them. Hence, although pedagogically interesting, slow gas expulsion is unlikely to play a role in bound cluster formation with age $\gtrsim 4$ Myr. Hence, it seems more likely that the few $10 \mathrm{~s}$ pc YMCs are ensembles of closely-located YMCs $(\sim 10$ s of them) forming low-mass cluster complexes, thus being younger, low-mass versions of "faint-fuzzy" objects (Brüns et al. 2009; Amaro-Seoane et al. 2014). Future observations of the light profiles of these objects would help to better understand their nature.

\section{Cluster formation through hierarchical mergers}

So far we have considered monolithic or in situ formation of star clusters. Given the substructured and filamentary conditions in molecular clouds in which stars form (see Sect. 1), it is plausible that YMCs may also arise from the sequential mergers of less massive sub-clusters (Longmore et al. 2014; Fujii et al. 2012; Smith et al. 2013; Banerjee \& Kroupa 2015a). 

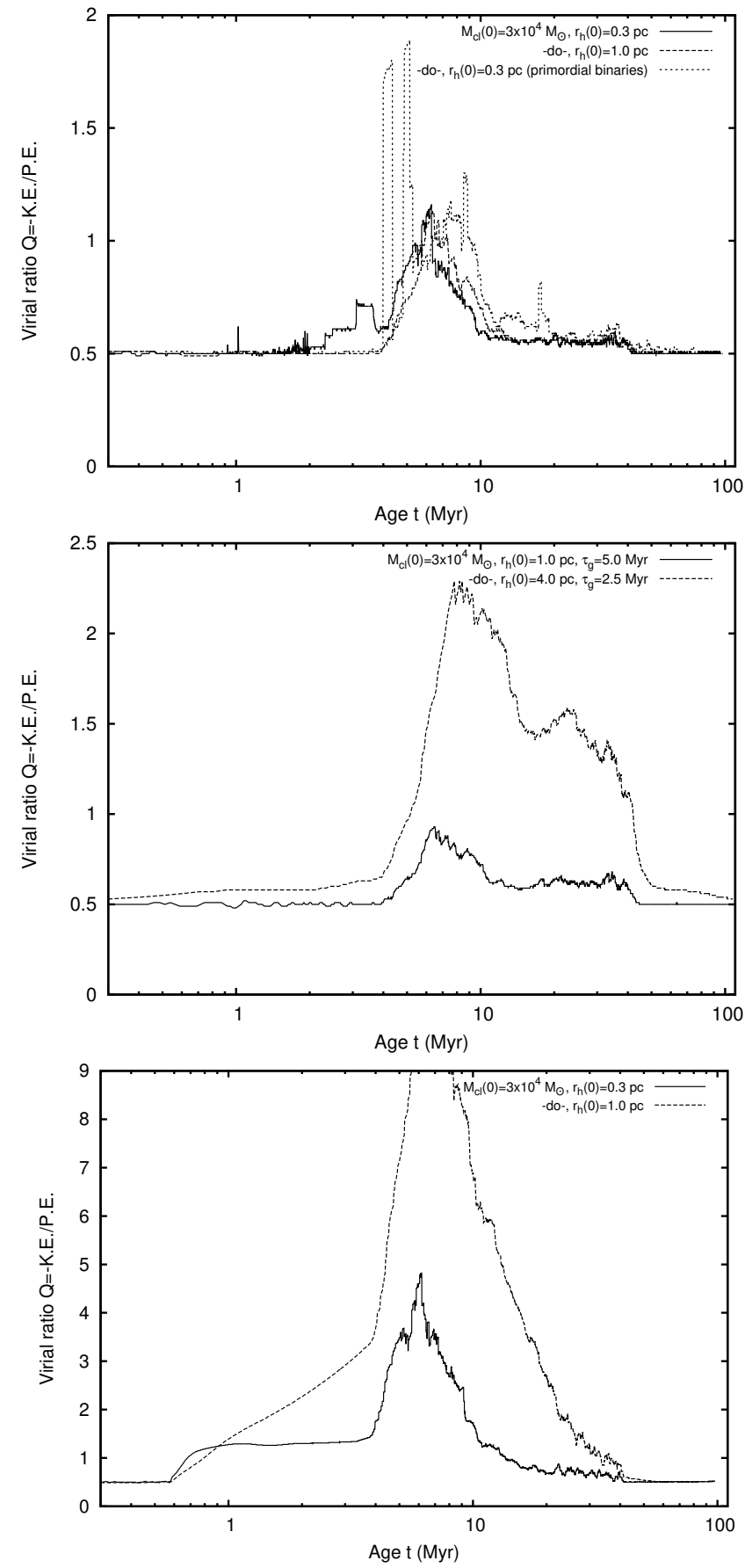

Fig. 9. Evolution of the virial ratio $Q$, i.e. the ratio of the total kinetic to potential energy of the bound cluster members for representative computed cluster models without gas expulsion (top panel) and those with placid (middle) and explosive (bottom) gas expulsion. For the cases without and with placid gas expulsion, $Q \approx 0.5$, i.e. the system is in dynamical equilibrium over most of the evolutionary times. In these two cases, $Q$ temporarily grows (i.e. the cluster becomes supervirial) after $t \approx 4 \mathrm{Myr}$ (which corresponds to a rapid expansion of the clusters' core) owing to substantial mass loss due to supernovae. This phase lasts $\approx 50$ Myr over which the BHs and the NSs form. The placid gas expulsion aids this non-dynamical-equilibrium expansion phase of the cluster resulting in larger growth of $Q$ (cf., middle and top pan$e l s)$. For explosive gas removal, a cluster expands in a super-virial manner from the beginning of gas expulsion $\left(\tau_{\mathrm{d}} \approx 0.6 \mathrm{Myr}\right)$ and becomes even more super-virial when the supernovae begin (bottom panel). Like the other cases, the system eventually returns to dynamical equilibrium when the supernova mass loss is quenched. As expected, in all the cases the growth of $Q$ is larger for the initially more extended cluster.
These sub-clusters, where the massive proto-stars preferably form and which would appear along the filaments and at filament junctions, would fall through the molecular cloud into the deepest part of the potential well where they would merge into a single proto-cluster. This overall picture is revealed in several recent hydrodynamic simulations of cluster-sized molecular clouds that include radiative stellar feedback.

In this scenario, the two following cases can be distinguished, namely (a) a starburst occurs over the free-fall time of the cloud so that the newly formed proto-stellar sub-clusters, which contribute to the formation of the YMC, and the gas fall in concurrently (see e.g. Bate \& Bonnell 2004; Bate 2009) and (b) the star-formation rate is diminished (e.g. due to stellar feedback and heating of the gas as in Dale et al. 2015) so that the ensemble of the contributing proto-subclusters forms over a timescale substantially longer than the free-fall time. Traditionally, case (a) is referred to as "coupled" star formation and case (b) as "decoupled". Coupled star formation would essentially lead to a single, monolithic embedded cluster after the merger phase, as studied in Sect. 3. On the other hand, decoupled star formation implies cluster assembling independent of the motion of the gas and over timescales longer than the free-fall time.

Note that if a cluster has to form and evolve from a young age (a few Myr, like the youngest Galactic YMCs), the sub-clusters should fall in from sufficiently close separation so that they can merge early enough. This is demonstrated in Banerjee \& Kroupa (2015a) for the case of the $\approx 1$ Myr-old NGC 3603 young cluster, for which the sub-clusters must merge from $\lesssim 2 \mathrm{pc}$. This implies that although star formation is often found to occur over $\gtrsim 10$ pc regions, only a part of the newly formed stellar structure can actually comprise a YMC. There is mounting observational evidence of young stellar and/or proto-stellar sub-clusters packed within pc-scale regions (e.g. Massi et al. 2015; Román-Zúniga et al. 2015). Furthermore, as demonstrated in Banerjee \& Kroupa (2015a), the "prompt" merger of several gas-filament-like compact sub-clusters produces a similarly compact cluster. Hence, as the calculations in Sect. 2.2 suggest, the presently observed YMC sizes would be unreachable for the newly assembled cluster via pure secular expansion. This is also true for the individual sub-clusters. Therefore, as demonstrated in Banerjee \& Kroupa (2015a), a post-merger explosive gas expulsion is instrumental in yielding YMCs that are similar to those we observe. On the other hand, if the sub-clusters are initially sufficiently apart that gas expulsion occurs in them individually prior to the merger (Fellhauer \& Kroupa 2005), then the likely outcome would be a highly diffuse, massive stellar association with substructures, and it may, as a whole, be super-virial or subvirial, for example Cyg-OB2.

\section{Discussions and conclusions}

While expansion of a star cluster driven by two-body relaxation, binary heating, and stellar mass loss is a fairly familiar phenomenon, its impact on shaping young star clusters remains a matter of debate. The calculations in Sect. 2.2 show that if star clusters preferably appear within the filamentary overdense structures of molecular clouds, adapting to their typical dimensions of $0.1-0.3 \mathrm{pc}$, then the self-driven secular expansion is generally insufficient for such clusters to reach the dimensions of observed YMCs. This is true if the cluster forms monolithically or via hierarchical mergers of closely located sub-clusters, and holds irrespective of the newborn cluster's mass. We note that the monolithic cluster or the sub-clusters should naturally adapt to the compact sizes of the filamentary and dense substructures 
inside molecular clouds. While formation of proto-stars outside the filaments, i.e. in rarer media, cannot be completely ruled out, both observations (e.g. Schneider et al. 2010, 2012; André et al. 2014) and hydrodynamic calculations (e.g. Klessen et al. 1998; Bate \& Bonnell 2004; Girichidis et al. 2011) suggest that the majority of the proto-stars must form within the compact dimensions of the dense gas filaments. A fraction of them can thereafter migrate to less dense parts of the local cloud via dynamical interactions.

The above direct $N$-body calculations naturally include an accurate treatment of two-body relaxation and dynamical encounters involving binaries. The other major processes that drive a cluster's expansion are stellar mass loss (until $\approx 50 \mathrm{Myr}$ ) and dynamical heating by compact remnants. As discussed in Sect. 2.2, despite the shortcomings of the implementation of stellar mass loss in NBODY7 (as such, in any $N$-body code at present), the computed secular expansion is nearly independent of the details of stellar winds and supernovae and serves as an upper limit. Also, while the computed models here begin with Plummer profiles, such a specific initial profile does not influence a cluster's overall expansion rate, as determined by $r_{\mathrm{eff}}(t)$, which is driven by the overall stellar mass loss and dynamical encounters in the inner part of the cluster. In all cases, a Plummer would be an appropriate initial profile since the molecular clouds' filaments are found to possess Plummer-like crosssections (Malinen et al. 2012). In the present calculations including primordial binaries, the massive $\mathrm{O}$-star binaries, which are efficient in energy generation through dynamical interactions, have a period distribution and binary fraction that is consistent with observations (see Sect. 2.1). This necessitates additional mechanisms that aid a young star cluster's expansion.

Having run out of the other possibilities, expulsion of residual gas seems to be the only process that evolves a newly hatched cluster from its compact configuration to the observed sizes of YMCs, as the calculations of Sect. 3 show. However, the widest (and oldest) $\sim 10$ pc-sized YMCs still seem difficult to achieve in this way (see Sect. 3.1), and they are more likely to be ensembles of several young clusters (see Sect. 3.2).

An important concern in the above line of argument is how YMCs and SSCs gather their total mass of $\gtrsim 10^{4} M_{\odot}$ within a few Myr, irrespective of their formation channels. This "massbudget problem" in the context of cluster assembly is, at present, essentially unsettled, both from observational and computational point of view. There is no clear example of such an amount of assembling mass in molecular clouds in our Galaxy. However, as discussed in the previous sections, the assembly phase of starburst clusters is short, $\lesssim 1$ Myr. Therefore, it would be rare to find a starburst cluster in its assembling phase in a normal gasrich disc galaxy like the Milky Way; one finds either widely distributed clouds or already assembled VYMCs. The chances of catching VYMCs and SSCs in their assembling phase would be higher in starburst galaxies where a much larger number of massive clusters are triggered. Indeed, recent ALMA observations of the Antennae Galaxy indicate still-forming, deeply embedded stellar systems of total mass exceeding $10^{7} M_{\odot}$, which are either monolithic systems or distributed over a few pc (Johnson 2015; Johnson et al. 2015). In order to better understand the conditions under which VYMCs and SSCs form, improved and more exhaustive observations of starburst galaxies are necessary.

Hydrodynamic simulations of molecular clouds cannot presently be done with such a large mass, keeping sufficient resolution at the same time. However, a recent work by Dale et al. (2015) involves low-resolution (mass resolution 10-100 $M_{\odot}$ ) smoothed-particle-hydrodynamics (SPH) calculations of massive $\left(10^{5}-10^{6} M_{\odot}\right)$, turbulent molecular clouds. These calculations include radiative feedback onto the gas from ionizing O/B stars, but have no magnetic fields. A highresolution (reaching the "opacity limit") SPH computation over such a mass scale and the inclusion of a magnetic field is technologically prohibitive at present. Dale et al. (2015) find subcluster formation at the deepest parts of the potential well where the SFE approaches $\approx 100 \%$. Furthermore, the gas is ionized and cleared from the neighbourhood of the sub-clusters, causing essentially gas-free mergers of the sub-clusters. This inhibits any mechanical effect of radiative gas blow-out on the merged cluster, as Dale et al. (2015) argue.

Notably, these simulations are too poorly resolved to reliably infer any SFE. Also, since the sub-clusters themselves are treated as sink particles, it is impossible to estimate the size of the final merged cluster. The sink-particle treatment of sub-clusters would also grossly overestimate the SFE at their locations. Highresolution SPH calculations (but for much less massive clouds, $\sim 100 M_{\odot}$ ), including radiative feedback and magnetic field (see Sect. 3 and references therein), obtain SFE $\lesssim 30 \%$. Hence, although the most massive set of molecular-gas SPH simulations to date, the treatment of gas in Dale et al. (2015) and its inferred role in assembling a cluster cannot be taken as conclusive. This limitation is essentially imposed by the present technology.

The treatment of gas is simplistic in the present work as well. However, it still captures the overall dynamical effect of gas blow-out on a newly-assembled cluster. We note that that a maximum localized SFE of $30 \%$ is assumed in the computations in Sect. 3, implying that in practice the impact of gas expulsion could be stronger. Also, the present arguments are based on details like the model clusters' profiles and their spatial scales are taken into account. The present approach necessitates a significant role of rapid explosive or near-explosive gas blow out that transforms infant clusters to observed profiles, in general. Notably, with $\approx 30 \%$ SFE, a $\approx 10^{5} M_{\odot}\left(10^{4} M_{\odot}\right)$ cluster leaves $\approx 80(30) \%$ of its mass bound (Brinkmann et al., in prep.) following an explosive gas expulsion from compact initial conditions. In other words, such a gas expulsion does not dissolve a YMC progenitor and retains a substantial fraction of it, in general agreement with Dale et al. (2015). As pointed out in Sects. 1 and 3 , the efficient violent relaxation, due to the high stellar density in massive compact proto-clusters, causes them to remain well bound (but to expand) even after $\approx 70 \%$ of their mass is expelled explosively. Such a short gas-expulsion phase is, of course, difficult to catch observationally. One such candidate is the RCW 38 cluster (DeRose et al. 2009), which is highly compact $\left(r_{\mathrm{h}} \approx 0.1 \mathrm{pc}\right)$ and is exposed only over its central region.

It may be argued that the observed YMCs are formed from $r_{\mathrm{h}}(0)>1 \mathrm{pc}$ initial configurations and with $S F E \approx 100 \%$, which would evolve to the observed YMCs with $r_{\text {eff }} \gtrsim 1 \mathrm{pc}$. From observations, we also know that $r_{\mathrm{h}}(0) \lesssim 0.3 \mathrm{pc}$ is common for infant clusters (André et al. 2011), implying that there should also be a population of gas-free clusters with ages $t>10 \mathrm{Myr}$ but $r_{\text {eff }} \lesssim 1$ pc, assuming again an SFE $\approx 100 \%$ (Fig. 3) since in more compact and dense environments the SFE is unlikely to decrease. No such bimodal population of exposed clusters is known, from which it follows that $r_{\mathrm{h}}(0) \lesssim 0.3 \mathrm{pc}$ and $S F E \approx 30 \%$ is the most likely birth condition for YMCs. Also, the observed $r_{\text {eff }}-t$ trend of the present-day YMCs may be consistent with their expansion due to stellar mass loss with a considerable scatter though, as in e.g. Ryon et al. (2015). However, this does not preclude an early gas-expulsion phase; once the surviving cluster, after the gas depletion, returns to (near) dynamical equilibrium (Banerjee \& Kroupa 2013), the expanded 
cluster continues to expand (cf. Fig. 6) owing to stellar mass loss and two-body relaxation processes (Sect. 2).

Notably, based on the velocity-space morphologies of gas clouds (Haworth et al. 2015) in the neighbourhood of several starburst clusters, some authors (Furukawa et al. 2009; Fukui et al. 2014, 2016) infer that these clusters (e.g. Westerlund 2, NGC 3603) form out of intense starbursts triggered during major cloud-cloud collisions. In this scenario, the residual gas is effectively removed (without the aid of stellar feedback) as the clouds cross and move away from each other. Such an interaction phase typically lasts for $\lesssim 1 \mathrm{Myr}$, implying that the cluster has to form monolithically or near monolithically (Banerjee \& Kroupa 2015a). In this case, the cluster formed in the colliding cloud would also expand explosively, unless the conversion from gas to star in the shocked region is nearly complete. As discussed in Sect. 3, both observations and highresolution SPH simulations, performed so far, seem to disfavour the latter condition. A thorough survey of the kinematics of gas around YMCs would help to reveal the role of the gas from which these clusters were born.

Acknowledgements. We thank the referee for constructive criticisms that led to substantial improvements of the description and discussions in the paper.

\section{References}

Aarseth, S. J. 2003, Gravitational N-Body Simulations (Cambridge University Press)

Aarseth, S. J. 2012, MNRAS, 422, 841

Amaro-Seoane, P., Konstantinidis, S., Freitag, M. D., et al. 2014, ApJ, 782, 97

André, P., Meńshchikov, A., Koenyves, V., et al. 2011, in Stellar Clusters \& Associations: A RIA Workshop on Gaia, eds. E. J. Alfaro Navarro, A. T. Gallego Calvente, \& M. R. Zapatero Osorio (Granada: IAA-CSIC), 321

André, P., Di Francesco, J., Ward-Thompson, D., et al. 2014, in Protostars and Planets VI, eds. H. Beuther, R. Klessen, C. Dullemond, \& Th. Henning, (Tucson: University of Arizona Press), 27

Bally, J., Walawender, J., \& Reipurth, B. 2012, AJ, 144, 143

Bally, J., Ginsburg, A., Silvia, D., \& Youngblood, A. 2015, A\&A, 579, A130

Banerjee, S. 2011 in Proceedings of the 25th Texas Symposium on Relativistic Astrophysics - TEXAS 2010 [arXiv: 1102.4614]

Banerjee, S., \& Kroupa, P. 2012, A\&A, 547, A23

Banerjee, S., \& Kroupa, P. 2013, ApJ, 764, 29

Banerjee, S., \& Kroupa, P. 2014, ApJ, 787, 158

Banerjee, S., \& Kroupa, P. 2015a, MNRAS, 447, 728

Banerjee, S., \& Kroupa, P. 2015b, in The Birth of Star Clusters, ed. S. W. Stahler (Springer-Verlag) [arXiv: 1512 .03074]

Banerjee, S., Kroupa, P., \& Oh. S. 2012, ApJ, 746, 15

Banerjee, S., Baumgardt, H., \& Kroupa, P. 2010, MNRAS, 402, 371

Bastian, N., \& Silva-Villa, E. 2013, MNRAS, 431, L122

Bate, M. R., 2009, MNRAS, 392, 590

Bate, M. R. 2012, MNRAS, 419, 3115

Bate, M. R., \& Bonnell, I. A. 2004, The Formation and Evolution of Massive Young Star Clusters, eds. H. J. G. L. M. Lamers, L. J., Smith, \& A. Nota, ASP Conf. Proc., 322, 289

Bate, M. R., Tricco, T. S., \& Price, D. J. 2014, MNRAS, 437, 77

Baumgardt, H., \& Kroupa, P., 2007, MNRAS, 380, 1589

Baumgardt, H., De Marchi, G., \& Kroupa, P. 2008, ApJ, 685, 247

Bik, A., Stolte, A., Gennaro, M., et al. 2014, A\&A, 561, A12

Boily, C., \& Kroupa, P. 2003a, MNRAS, 338, 665

Boily, C., \& Kroupa, P. 2003b, MNRAS, 338, 673

Brüns, R. C., Kroupa, P., \& Fellhauer, M. 2009, ApJ, 702, 1268

Dale, J. E., Ercolano, B., \& Bonnell, I. A. 2015, MNRAS, 451, 5506

de Mink, S. E., Pols, O. R., Langer, N., \& Izzard, R. G. 2009, A\&A, 507, L1

DeRose, K. L., Bourke, T. L., Gutermuth, R. A., et al. 2009, AJ, 138, 33
Duarte-Cabral, A., Dobbs, C. L., Peretto, N., et al. 2011, A\&A, 528, A50 Elmegreen, B. G. 1983, MNRAS, 203, 1011

Feigelson, E. D., \& Townsley, L. K. 2008, ApJ, 673, 354

Fellhauer, M., \& Kroupa, P. 2005, ApJ, 630, 879

Fujii, M. S., Saitoh, T. R., \& Portegies Zwart, S. F. 2012, ApJ, 753, 85

Fukui, Y., Ohama, A., Hanaoka, N., et al. 2014, ApJ, 780, 36

Fukui, Y., Torii, K., Ohama, A., et al. 2016, ApJ, 820, 26

Furukawa, N., Dawson, J. R., Ohama, A., et al. 2009, ApJ, 696, L11

Gieles, M., Moeckel N., \& Clarke, C. J. 2012, MNRAS, 426, L11

Girichidis, P., Federrath, C., Banerjee, R., \& Klessen, R. S. 2011, MNRAS, 413, 2741

Girichidis, P., Federrath, C., Banerjee, R., \& Klessen, R. S. 2012, MNRAS, 420, 613

Gratton, R. G., Carretta, E., \& Bragaglia, A. 2012, A\&ARv, 20, 50

Haworth, T. J., Tasker, E. J., Fukui, Y., et al. 2015, MNRAS, 450, 10

Heggie, D. C. 1975, MNRAS, 173, 729

Heggie, D. C., \& Hut, P. 2003, The Gravitational Millon-Body Problem: A Multidisciplinary Approach to Star Cluster Dynamics (Cambridge, UK: Cambridge University Press)

Hènon, M. 1965, A\&A, 28, 62

Hollyhead, K., Bastian, N., Adamo, A., et al. 2015, MNRAS, 449, 1106

Hurley, J. R., Pols, O. R., \& Tout, C. A. 2000, MNRAS, 315, 543

Hurley, J. R., Tout, C. A., \& Pols, O. R. 2002, MNRAS, 329, 897

Johnson, K. 2015, IAU GA Meeting 29, id. 2257770

Johnson, K. E., Leroy, A. K., Indebetouw, R., et al. 2015, ApJ, 806, 35

Klessen, R. S., Burkert, A., \& Bate, M. R. 1998, ApJ, 501, L205

Kroupa, P. 1995, MNRAS, 277, 1507

Kroupa, P. 2001, MNRAS, 322, 231

Kroupa, P. 2008, in Initial Conditions for Star Clusters. eds. S. J. Aarseth, C. A

Tout, \& R. A. Mardling, Lect. Notes Phys. (Berlin: Springer-Verlag), 760, 181

Kroupa, P., Aarseth, S., \& Hurley, J. 2001, MNRAS, 321, 699

Kruijssen, J. M. D., Maschberger, T., Moeckel, N., et al. 2012, MNRAS, 419, 841

Lada, C. J., \& Lada, E. A. 2003, ARA\&A, 41, 57

Longmore, S. N., Kruijssen, J. M. D., Bastian, N., et al. 2014, in Protostars and Planets VI, eds. H. Beuther, R. Klessen, C. Dullemond, \& Th. Henning (Tucson: University of Arizona Press), 291

Machida, M. N., \& Matsumoto, T. 2012, MNRAS, 421, 588

Mackey, A. D., \& Gilmore, G. F. 2003a, MNRAS, 338, 85

Mackey, A. D., \& Gilmore, G. F. 2003b, MNRAS, 338, 120

Mackey, A. D., Wilkinson, M. I., Davies, M. B., \& Gilmore, G. F. 2008, MNRAS, 386, 65

Malinen, J., Juvela, M., Rawlings, M. G., et al. 2012, A\&A, 544, A50

Marks, M., \& Kroupa, P. 2012, A\&A, 543, A8

Massi, F., Giannetti, A., \& di Carlo, E. 2015, A\&A, 573, A95

Messineo, M., Clark, J. S., Figer, D. F., et al. 2015, ApJ, 805, 110

Morscher, M., Umbreit, S., Farr, W. M., \& Rasio, F. A. 2013, ApJ, 763, L15

Oh, S., Kroupa, P., \& Pflamm-Altenburg, J. 2015, ApJ, 805, 92

Pfalzner, S. 2009, A\&A, 498, L37

Pfalzner, S., \& Kaczmarek, T. 2013, A\&A, 559, A38

Pfalzner, S., Parmentier, G., Steinhausen, M., et al. 2014, ApJ, 794, 147

Pflamm-Altenburg, J., \& Kroupa, P. 2009, MNRAS, 397, 488

Plummer, H. C. 1911, MNRAS, 71, 460

Portegies Zwart, S. F., McMillan, S. L. W., \& Gieles, M. 2010, ARA\&A, 48, 431

Rathborne, J. M., Longmore, S. N., Jackson, J. M., et al. 2015, ApJ, 802, 125

Renaud, F., Bournaud, F., \& Duc, P-A. 2015, MNRAS, 446, 2038

Román-Zúniga, C. G., Ybarra, J., Megias, G., et al. 2015, AJ, 150, 80

Ryon, J. E., Bastian, N., Adamo, A., et al. 2015, MNRAS, 452, 525

Sana, H., \& Evans, C. J. 2011, in Active OB Stars: Structure, Evolution, Mass Loss, and Critical Limits, eds. C. Neiner, G. Wade, G. Meynet, \& G. Peters (Cambridge: Cambridge Univ. Press), IAU Symp., 272, 474

Schneider, N., Csengeri, T., Bontemps, S., et al. 2010, A\&A, 520, A49

Schneider, N., Csengeri, T., Hennemann, M., et al. 2012, A\&A, 540, L11

Smith, R., Goodwin, S., Fellhauer, M., \& Assmann, P. 2013, MNRAS, 428, 1303

Spitzer, L. Jr. 1987, Dynamical Evolution of Globular Clusters (Princeton University Press)

Tafalla, M., \& Hacar, A. 2014, A\&A, 574, A104

Tapia, M., Roth, M., Bohigas, J., et al. 2011, MNRAS, 416, 2163

Traficante, A., Fuller, G. A., Peretto, N., et al. 2015, MNRAS, 451, 3089

Weidner, C., \& Kroupa, P. 2004, MNRAS, 348, 187

Weidner, C., Kroupa, P., \& Pflamm-Altenburg, J. 2013, MNRAS, 434, 84 